YITP-SB-06-30

\title{
Hybrid Formalism, Supersymmetry Reduction, and Ramond-Ramond Fluxes
}

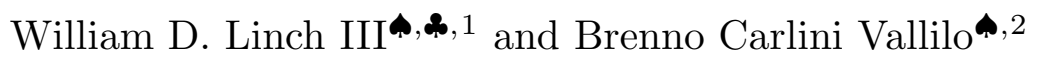 \\ ^ C.N. Yang Institute for Theoretical Physics and Department of Mathematics \\ SUNY, Stony Brook, NY 11794-3840, USA
}

The supersymmetric hybrid formalism for Type II strings is used to study partial supersymmetry breaking in four and three dimensions. We use worldsheet techniques to derive effects of internal Ramond-Ramond fluxes such as torsions, superpotentials and warping.

July 2006

1 E-Mail: wdlinch3 (at) math.sunysb.edu

2 E-Mail: vallilo (at) insti.physics.sunysb.edu 


\section{Contents}

1. Introduction . . . . . . . . . . . . . . . . . . . . . . . . . . . . . 1

2. Hybrid Formalism in $d=4$. . . . . . . . . . . . . . . . . . . . . . . . . . . . . . . . .

2.1. Action and Symmetries . . . . . . . . . . . . . . . . . . . . . . . . . . . 4

2.2. Coupling to $c=9, N=2 \mathrm{CFTs}$. . . . . . . . . . . . . . . . . . . . . . . 6

2.3. Physical States . . . . . . . . . . . . . . . . . . . . . . 8

2.4. Deformations of the Action . . . . . . . . . . . . . . . . . . . . . . . . . 11

2.5. Supersymmetric Amplitudes . . . . . . . . . . . . . . . . . . . . . . . . . 12

3. Dimensional Reduction, Quotients and $G_{2}$ Holonomy . . . . . . . . . . . . . . . . 14

3.1. The Superspace ${ }^{N} d={ }^{2} 4$ and Its Reductions . . . . . . . . . . . . . . . . . . 14

3.2. $G_{2}$ Structure and $\mathbb{Z}_{2}$ Quotient . . . . . . . . . . . . . . . . . . . . . . . . 17

4. Internal Ramond-Ramond Fluxes and Deformations . . . . . . . . . . . . . . . . 19

5 . Conclusions and Further Directions . . . . . . . . . . . . . . . . . . . . . . . 25

Appendix A. Hybrid Compactified on $S^{1}$ : Supersymmetric Operators in $d=3$. . . . . . 26

\section{Introduction}

String theory in background fields, especially Ramond-Ramond backgrounds, is one of the main themes in the field. It has become clear that in order to gain a better understanding of many phenomena of recent interest we cannot avoid studying RR flux in the stringy regime.

For more than ten years, Berkovits and collaborators have been developing a series of super-Poincaré covariant formalisms for the superstring. In two [1], four [2], and six [3, [1] dimensions the hybrid formalism is obtained from a field redefinition of the RNS superstring and has an enhanced symmetry algebra on the worldsheet, namely $N=4$, $\widehat{c}=2$ superconformal symmetry. Although they share a common structure, these algebras have different expressions in terms of the fundamental fields in each dimension and all of them have chiral bosons as fundamental worldsheet fields. It is natural to ask whether there is a general principle behind these formulations other than the underlying relation with the RNS superstring.

The minimal3 pure spinor formalism [6] holds a special place among all these formalisms; it has no superconformal symmetry and no scalar ghosts. Instead, it has a set bosonic ghosts in the spinor representation of the Lorenz group satisfying the pure spinor

3 Here we are using the nomenclature introduced in [5]. 
constraint. Similarly, in the covariant approach [4] for the six-dimensional hybrid formalism one is forcedt to introduce a set of unconstrained spinor ghosts and a BRST charge $Q=\oint u^{\alpha} \mathcal{D}_{\alpha}$ where $\mathcal{D}_{\alpha}$ is the projective superspace derivative. These new ghosts and BRST charge have nothing to do with the underlying RNS formalism and are required to have manifest supersymmetry in $d=6$. In this case the bosonic spinor ghosts do not have to satisfy any constraint since the projective superspace derivative commutes with itself. (If one tries to increase the number of supersymmetries again one finds that it is not possible to construct a set of commuting supersymmetric derivatives, and thus the pure spinor constraint is necessary.) Nevertheless these new ghosts interact with the original variables and must appear in the vertex operators. We note that this case straddles the usual hybrid formalism and the pure spinor superstring. It would be very interesting to find a deeper relation between them, possibly through the superconformal extension of the minimal pure spinor [5].

Although supersymmetry is one of the main ingredients in string theory, superspace techniques have historically always played a peripheral role. The first reason is that until recently there was no quantizable formalism for the superstring with all supersymmetries

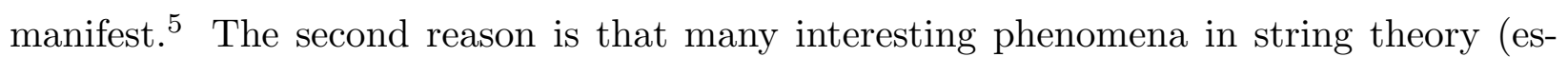
pecially the construction of models which resemble the observed particle physics) appear after breaking some supersymmetry. For example, ten-dimensional Type I superspace has encoded within itself the $N=1$ four-dimensional superspace but from the ten-dimensional point of view it is difficult to see how holomorphicity and non-renormalization theorems appear. This has become clear in superstring field theory [9,10] where chiral and anti-chiral $F$-terms appear, but the procedure is only possible after breaking some manifest Lorentz symmetry. Finally, one can argue that the usefulness of superspace in higher dimensions is restricted by our lack of understanding of it's off-shell structure.

Breaking supersymmetry in higher-dimensional theories is usually done in components and the superspace, if it is introduced at all, only appears in the very beginning and end of the analysis. Furthermore, the Grassmann coordinates related to higher supersymmetries are simply set to zero by hand. In a supersymmetric covariant formalism it is not consistent

4 This procedure was described in footnotes in [6].

5 In two interesting papers Lee and Siegel [0],8] introduced a new formalism for the superstring based on the usual GS formalism but with a consistent BRST charge built on an infinite pyramid of ghosts in addition to the usual $(b, c)$ system of the bosonic string. Scattering amplitudes are computed using very simple rules and its application to other problems seems promising. 
to do this since the superspace coordinates are part of the conformal field theory describing the superstring; they have to satisfy consistency conditions like vanishing of central charge and are essential for the worldsheet symmetries. In order to study compactifications of covariant formalisms we need a method to dimensionally reduce and break supersymmetry keeping all of the original superspace coordinates.6 In this paper we take a small step in this direction by studying the standard four-dimensional hybrid superstring in backgrounds with reduced symmetry such as Type IIA on a $G_{2}$ holonomy manifold and with RamondRamond flux in the internal space.

The hybrid formalism has been used previously to study strings in RR backgrounds, for example, in the case of $A d S_{3} \times S^{3}$ [12], $A d S_{2} \times S^{2}$ [13], the $C$-deformation [14] and noncommutative superspace [15]. In all of these examples the RR flux considered was in the uncompactified sector of space-time, with no contributions coming from the internal manifold. The relation of internal RR fluxes with auxiliary fields in the four-dimensional supersymmetric multiplets first appeared in [16] and was discussed further in [17] and [18]. Compactifications including fluxes have attracted a lot of attention in recent years due to their applications to the problem of moduli stabilization (see [19] and references therein).

We hope that the superspace reduction introduced in this paper can be extended to the higher-dimensional versions of the hybrid formalism and possibly to the (minimal) pure spinor formalism. This type of superspace reduction could also be useful for finding the relation with the pure spinor superstring; there is the possibility that the pure spinor formalism is the generating formalism for all covariant formalisms in lower dimensions. This, in turn, might help to better understand the new superconformal description of [5].

Another application of the present work is to to study $G_{2}$ holonomy compactifications, at least in the case where the $G_{2}$ is of the form $\left(\mathrm{CY}_{3} \times S^{1}\right) / \mathbb{Z}_{2}$. If we start with M-theory we have a four-dimensional effective field theory with $N=1$ supersymmetry and with the appropriate manifold we can obtain $N=1$ super YM [20]. Since we do not have a (covariant) microscopic description of M-theory, we can further compactify the theory on a circle and use the duality with Type IIA to address stringy questions. Yet another application is the conjectured relation between Hitchin functionals in seven dimensions and topological strings on Calabi-Yau manifolds [21]. Since, in the hybrid formalism, there is

6 The inverse problem, i.e. the use of standard four-dimensional $N=1$ superspace to describe higher-dimensional theories and those with more supersymmetry, has been widely applied in the literature. For a relatively recent discussion with applications to phenomenology see 11. 
a well-defined way to compute general supersymmetric amplitudes, it is possible to use the covariant description of the present paper to calculate amplitudes in backgrounds of the form $\left(\mathrm{CY}_{3} \times S^{1}\right) / \mathbb{Z}_{2}$ and see what terms topological amplitudes are computing in the three-dimensional effective action.

This paper is organized as follows. In the next section we will review the fourdimensional hybrid formalism, stressing features which are going to be useful in the subsequent sections and have not heretofore appeared in the literature, such as alternative descriptions of vertex operators and amplitudes in the compactification-dependent sector. The three-dimensional $N=4,2$ and 1 superspace will be described in section 3 . A convenient way to reduce the number of supersymmetries without leaving the original superspace will be introduced. Multiplets with various amounts of supersymmetry will be described. We then show how the hybrid superstring in four dimensions can be used to describe string theories in lower dimensions without changing the number of fundamental fields in the formalism. We then apply these methods to give a supersymmetric description of Type IIA on $\left(\mathrm{CY}_{3} \times S^{1}\right) / \mathbb{Z}_{2}$. In section 4 , we discuss effects of Ramond-Ramond fields in four dimensions (which is easily adapted to the case of three dimensions using the results of section 3). In the concluding section we summarize the work and comment on future applications to problems of current interest. Finally, we include for completeness an appendix with the hybrid formalism reduced on $S^{1}$ in the conventions of section 3 .

\section{Hybrid Formalism in $d=4$}

In this section we review basic aspects of the hybrid formalism in $d=4$ [2]. Besides setting up definitions and notations, we comment on aspects of the formalism which have not appeared previously in the literature such as supersymmetric amplitudes in the compactification sector.

\subsection{Action and Symmetries}

The original formulation of the hybrid superstring is as a field redefinition of the RNS variables compactified on a Calabi-Yau background [2]. In its final form there is a complete decoupling between the four-dimensional flat space and the Calabi-Yau background. The fundamental variables of the $d=4$ (closed string) hybrid formalism are the $N=2$ super-

space coordinates $\left(x, \theta_{L}, \bar{\theta}_{L}, \theta_{R}, \bar{\theta}_{R}\right)$, the conjugate momenta for the fermionic coordinates $\left(p_{L}, \bar{p}_{L}, p_{R}, \bar{p}_{R}\right)$ and two chiral bosons $\left(\rho_{L}, \rho_{R}\right)$. 
The action is

$$
S_{\text {hybrid }}=\int d^{2} z\left[\partial_{L} x^{m} \partial_{R} x_{m}+p_{L \alpha} \partial_{R} \theta_{L}^{\alpha}+\bar{p}_{L \dot{\alpha}} \partial_{R} \bar{\theta}_{L}^{\dot{\alpha}}+p_{R \alpha} \partial_{L} \theta_{R}^{\alpha}+\bar{p}_{R \dot{\alpha}} \partial_{L} \bar{\theta}_{R}^{\dot{\alpha}}\right]+S_{\text {chiral }} \text {, }
$$

where $S_{\text {chiral }}$ is the action for the chiral bosons. The fundamental OPE's are:

$$
\begin{gathered}
x^{m}(z) x^{n}(w) \rightarrow \eta^{m n} \ln |z-w|^{2}, \\
p_{L \alpha}(y) \theta_{L}^{\beta}(z) \rightarrow \frac{\delta_{\alpha}^{\beta}}{y-z}, \quad \bar{p}_{L \dot{\alpha}}(y) \bar{\theta}_{L}^{\dot{\beta}}(z) \rightarrow \frac{\delta_{\dot{\alpha}}^{\dot{\beta}}}{y-z}, \\
p_{R \alpha}(y) \theta_{R}^{\beta}(z) \rightarrow \frac{\delta_{\alpha}^{\beta}}{\bar{y}-\bar{z}}, \quad \bar{p}_{R \dot{\alpha}}(y) \bar{\theta}_{R}^{\dot{\beta}}(z) \rightarrow \frac{\delta_{\dot{\alpha}}^{\dot{\beta}}}{\bar{y}-\bar{z}}, \\
\rho_{L}(z) \rho_{L}(w) \rightarrow-\ln (z-w), \quad \rho_{R}(z) \rho_{R}(w) \rightarrow-\ln (\bar{z}-\bar{w}) .
\end{gathered}
$$

The last line shows that the chiral bosons are time-like and hence cannot be fermionized. Furthermore, they are space-time scalars.

The action (2.1) is supersymmetric, and the corresponding supercharges are

$$
\begin{aligned}
q_{L \alpha} & =\oint d z\left[p_{L \alpha}-\frac{i}{2} \bar{\theta}_{L}^{\dot{\alpha}} \partial_{L} x_{\alpha \dot{\alpha}}-\frac{1}{8}\left(\bar{\theta}_{L}\right)^{2} \partial_{L} \theta_{L \alpha}\right], \\
\bar{q}_{L \dot{\alpha}} & =\oint d z\left[\bar{p}_{L \dot{\alpha}}-\frac{i}{2} \theta^{\alpha}{ }_{L} \partial_{L} x_{\alpha \dot{\alpha}}-\frac{1}{8}\left(\theta_{L}\right)^{2} \partial_{L} \bar{\theta}_{L} \dot{\alpha}\right], \\
q_{R \alpha} & =\oint d z\left[p_{R \alpha}-\frac{i}{2} \bar{\theta}_{R}^{\dot{\alpha}} \partial_{R} x_{\alpha \dot{\alpha}}-\frac{1}{8}\left(\bar{\theta}_{R}\right)^{2} \partial_{R} \theta_{R \alpha}\right], \\
\bar{q}_{R \dot{\alpha}} & =\oint d z\left[\bar{p}_{R \dot{\alpha}}-\frac{i}{2} \theta^{\alpha}{ }_{R} \partial_{R} x_{\alpha \dot{\alpha}}-\frac{1}{8}\left(\theta_{R}\right)^{2} \partial_{R} \bar{\theta}_{R \dot{\alpha}}\right] .
\end{aligned}
$$

There is a set of operators which commutes with the charges (2.3) (and with their right-moving counterparts):

$$
\begin{gathered}
d_{L \alpha}=p_{L \alpha}+\frac{i}{2} \bar{\theta}_{L}^{\dot{\alpha}} \partial x_{\alpha \dot{\alpha}}-\frac{1}{4}\left(\bar{\theta}_{L}\right)^{2} \partial \theta_{L \alpha}+\frac{1}{8} \theta_{L \alpha} \partial\left(\bar{\theta}_{L}\right)^{2}, \\
\bar{d}_{L \dot{\alpha}}=\bar{p}_{L \dot{\alpha}}+\frac{i}{2} \theta_{L}^{\alpha} \partial x_{\alpha \dot{\alpha}}-\frac{1}{4}\left(\theta_{L}\right)^{2} \partial \bar{\theta}_{L \dot{\alpha}}+\frac{1}{8} \bar{\theta}_{L \dot{\alpha}} \partial\left(\theta_{L}\right)^{2}, \\
\Pi_{L}^{m}=\partial_{L} x^{m}-\frac{i}{2} \sigma_{\alpha \dot{\alpha}}^{m}\left(\theta_{L}^{\alpha} \partial_{L} \bar{\theta}_{L}^{\dot{\alpha}}+\bar{\theta}_{L}^{\dot{\alpha}} \partial_{L} \theta_{L}^{\alpha}\right),
\end{gathered}
$$

and similarly for the right moving sector. Here $x_{\alpha \dot{\alpha}}=x_{m} \sigma_{\alpha \dot{\alpha}}^{m}$. These operators realize the following algebra

$$
d_{L \alpha}(y) \bar{d}_{L \dot{\alpha}}(z) \rightarrow i \frac{\Pi_{L \alpha \dot{\alpha}}}{y-z}, \quad d_{L \alpha}(y) d_{L \beta}(z) \rightarrow \text { regular, }, \quad \bar{d}_{L \dot{\alpha}}(y) \bar{d}_{L \dot{\beta}}(z) \rightarrow \text { regular },
$$




$$
\begin{gathered}
d_{L \alpha}(y) \partial_{L} \theta_{L}^{\beta}(z) \rightarrow \frac{\delta_{\alpha}^{\beta}}{(y-z)^{2}}, \quad \bar{d}_{L \dot{\alpha}}(y) \partial_{L} \bar{\theta}_{L}^{\dot{\beta}}(z) \rightarrow \frac{\delta_{\dot{\alpha}}^{\dot{\beta}}}{(y-z)^{2}}, \\
d_{L \alpha}(y) \Pi_{L}^{m}(z) \rightarrow-i \frac{\sigma_{\alpha \dot{\alpha}}^{m} \partial_{L} \bar{\theta}_{L}^{\dot{\alpha}}}{y-z}, \quad \bar{d}_{L \dot{\alpha}}(y) \Pi_{L}^{m}(z) \rightarrow-i \frac{\sigma_{\alpha \dot{\alpha}}^{m} \partial_{L} \theta_{L}^{\alpha}}{y-z}, \\
\Pi_{L}^{m}(z) \Pi_{L}^{n}(z) \rightarrow-\frac{\eta^{m n}}{(y-z)^{2}} .
\end{gathered}
$$

Although it is not manifest, the action (2.1) in invariant under a non-linear $N=(2,2)$ superconformal transformation which is generated by

$$
\begin{gathered}
T_{L}=-\frac{1}{2} \partial_{L} x^{m} \partial_{L} x_{m}-p_{L \alpha} \partial_{L} \theta_{L}^{\alpha}-\bar{p}_{L \dot{\alpha}} \partial_{L} \bar{\theta}_{L}^{\dot{\alpha}}-\frac{1}{2} \partial_{L} \rho_{L} \partial_{L} \rho_{L}, \\
G_{L}^{+}=e^{\rho_{L}}\left(d_{L}\right)^{2}, \quad G_{L}^{-}=e^{-\rho_{L}}\left(\bar{d}_{L}\right)^{2}, \quad J_{L}=-\partial_{L} \rho_{L},
\end{gathered}
$$

again, together with the right-moving counterpart.

\subsection{Coupling to $c=9, N=2$ CFTs}

We can couple this $c=-3, N=2$ CFT to any $N=2$ CFT. Consistency of an $N=2$ superstring theory requires that the total central charge be $c=6$. This is the familiar condition for the standard critical $N=2$ string, after the introduction of the ghost sector. In the hybrid formalism this condition is better seen as a requirement to admit an $N=4$ topological description [3] in which no additional superconformal ghosts are needed. The $N=4$ formalism is suitable for defining scattering amplitudes and a string field theory action in the case of open strings. We will introduce some of its properties when needed.

There is the possibility of coupling the hybrid variables to a more supersymmetric CFT. This means that there are some space-time supersymmetries that are not linearly realized in the fundamental variables, and the hybrid description is not the most economical. The trivial example is the six-dimensional torus. Nevertheless, this example is very useful to compute exact answers in the CFT.

Given a $c=9, N=2$ CFT with left-moving generators $\left(\mathcal{T}_{L}, \mathcal{G}_{L}^{+}, \mathcal{G}_{L}^{-}, \mathcal{J}_{L}\right)$ a consistent string theory has action

$$
S=S_{\text {hybrid }}+S_{c=9}
$$

and $c=6, N=2$ generators

$$
T_{L}=-\frac{1}{2} \partial_{L} x^{m} \partial_{L} x_{m}-p_{L \alpha} \partial_{L} \theta_{L}^{\alpha}-\bar{p}_{L \dot{\alpha}} \partial_{L} \bar{\theta}_{L}^{\dot{\alpha}}-\frac{1}{2} \partial_{L} \rho_{L} \partial_{L} \rho_{L}+\mathcal{T}_{L},
$$




$$
\begin{gathered}
G_{L}^{+}=e^{\rho_{L}}\left(d_{L}\right)^{2}+\mathcal{G}_{L}^{+}, \quad G_{L}^{-}=e^{-\rho_{L}}\left(\bar{d}_{L}\right)^{2}+\mathcal{G}_{L}^{-}, \\
J_{L}=-\partial_{L} \rho_{L}+\mathcal{J}_{L} .
\end{gathered}
$$

The right-moving sector of the algebra is determined by the choice of Type IIA or Type IIB superstring. A consistent convention for the present work is the following 7

$$
\begin{array}{ll}
\text { Type IIA : } G_{R}^{+}=e^{\rho_{R}}\left(\bar{d}_{R}\right)^{2}+\mathcal{G}_{R}^{-}, \quad G_{R}^{-}=e^{-\rho_{R}}\left(d_{R}\right)^{2}+\mathcal{G}_{R}^{+}, \quad J_{R}=-\partial_{R} \rho_{R}-\mathcal{J}_{R} \\
\text { Type IIB : } G_{R}^{+}=e^{\rho_{R}}\left(d_{R}\right)^{2}+\mathcal{G}_{R}^{-}, \quad G_{R}^{-}=e^{-\rho_{R}}\left(\bar{d}_{R}\right)^{2}+\mathcal{G}_{R}^{+}, & J_{R}=-\partial_{R} \rho_{R}-\mathcal{J}_{R}
\end{array}
$$

The change in the energy-momentum tensor is given simply by switching $L \rightarrow R$ in derivatives and fields.

Since now the central charge is $c=6$, this system defines a critical $N=2$ superstring. One could now add superconformal ghosts and perform standard BRST quantization to define physical states and amplitudes but we will show that this is not necessary.

In the superconformal field theory of a Calabi-Yau 3-fold background we have, in addition to the usual superconformal algebra, a second superconformal algebra that does not commute with the first. We will call these generators

$$
\tilde{t}_{L}=\frac{1}{6} \mathcal{J}_{L}^{2}, \quad \tilde{g}_{L}^{+}=\frac{1}{\sqrt{3}} \Omega_{L}^{+}=e^{\mathcal{H}_{L}}, \quad \tilde{g}_{L}^{-}=\frac{1}{\sqrt{3}} \Omega_{L}^{-}=\frac{1}{\sqrt{3}} e^{-\mathcal{H}_{L}}, \quad \tilde{j}=\frac{1}{3} \mathcal{J}_{L},
$$

where $\partial_{L} \mathcal{H}_{L}=\mathcal{J}_{L}$ and $\Omega_{L}^{+}$and $\Omega_{L}^{-}$are holomorphic chiral and anti-chiral fields with charge 3 and -3 of the original superconformal algebra. They can be written in terms of the holomorphic $\Omega_{I J K}$ and anti-holomorphic $\bar{\Omega}_{\overline{I J K}} 3$-form on the Calabi-Yau respectively. The same applies to the right-moving sector and together these operators form an important part of the $N=4$ superconformal algebra.

To construct the extended superconformal algebra [3], we note that

$$
J_{L}=-\partial_{L} \rho_{L}+\mathcal{J}_{L}, \quad J_{L}^{++}=e^{-\rho_{L}} \Omega_{L}^{+}, \quad J_{L}^{--}=e^{+\rho_{L}} \Omega_{L}^{-},
$$

form an $s u(2)$ current algebra. With these operators we can generate two new superconformal operators

$$
\begin{gathered}
\tilde{G}_{L}^{+}=J_{L}^{++}\left(G_{L}^{-}\right)=\Omega_{L}^{+} e^{-2 \rho_{L}}\left(\bar{d}_{L}\right)^{2}+e^{-\rho_{L}} \Omega_{L}^{+}\left(\mathcal{G}_{L}^{-}\right), \\
\tilde{G}_{L}^{-}=J_{L}^{--}\left(G_{L}^{+}\right)=\Omega_{L}^{-} e^{2 \rho_{L}}\left(d_{L}\right)^{2}+e^{\rho_{L}} \Omega_{L}^{-}\left(G_{L}^{+}\right),
\end{gathered}
$$

The action of $\left(J_{L}^{++}, J_{L}^{--}\right)$on all other supercharges vanishes. A similar construction works in the right moving sector, but one should mind the conventions expressed in (2.8) and (2.9). The constraints $\left\{T_{L, R}, G_{L, R}^{ \pm}, \tilde{G}_{L, R}^{ \pm}, J_{L, R}, J_{L, R}^{ \pm \pm}\right\}$generate the required $N=4$ algebra.

7 This differs from the conventions in [22]. 


\subsection{Physical States}

We define physical states as primary fields of the algebra (2.7). Due to the large worldsheet symmetry algebra, vertex operators can be written in many equivalent ways. For every physical state, there is an infinite number of vertex operators representing it [3]. This large degeneracy is reminiscent of picture changing in the RNS formalism. Depending on the application, some choices have proven to be more useful than others. We are going to introduce some of them, explaining when each choice is suitable.

Massless vertex operators are constructed using neutral operators of conformal dimension zero times a function of the zero-modes of $\left(x^{m}, \theta_{L}^{\alpha}, \bar{\theta}_{L}^{\dot{\alpha}}, \theta_{R}^{\dot{\alpha}}, \bar{\theta}_{R}^{\dot{\alpha}}\right)$. In the simplest case the operator of conformal dimension zero is the identity operator 1 , and the vertex operator is just $V=U\left(x^{m}, \theta_{L}^{\alpha}, \bar{\theta}_{L}^{\dot{\alpha}}, \theta_{R}^{\dot{\alpha}}, \bar{\theta}_{R}^{\dot{\alpha}}\right) \times 1 . V$ is a primary field of conformal weight zero if

$$
\left(T_{L}\right)_{0} V=\left(G_{L}^{+}\right)_{\frac{1}{2}} V=\left(G_{L}^{-}\right)_{\frac{1}{2}} V=0
$$

together with the right-moving counterpart. Here $\mathcal{O}_{n} \mathcal{A}$ means the pole of order $h_{\mathcal{O}}+n$ in the OPE of $\mathcal{O}$ and $\mathcal{A}$ where $h_{\mathcal{O}}$ is the conformal weight of $\mathcal{O}$. Using the algebra (2.7) we have

$$
\nabla_{L}^{2} U=\bar{\nabla}_{L}^{2} U=\nabla_{R}^{2} U=\bar{\nabla}_{R}^{2} U=\square_{4} U=0
$$

where the $\nabla$ are the superspace covariant derivatives. These equations imply polarization and mass shell conditions for the superfield $U$ and it can be shown that $U$ is the prepotential for $N=2$ supergravity plus a tensor multiplet in a supersymmetric gauge. It is a general feature of the hybrid formalism that prepotentials (vs. potential or strength superfields) appear in the unintegrated vertex operators.

In principle one could also consider $U$ to be a real function of the Calabi-Yau coordinates $\left(y^{I}, \bar{y}^{\bar{I}}\right)$. We will require that this function is smooth so that it does not depend on the cohomology of the $\mathrm{CY}_{3}$. We will see that if there are NSNS and RR fluxes in the Calabi-Yau, $U$ will have such non-trivial $\left(y^{I}, \bar{y}^{\bar{I}}\right)$ dependence.

The other basic primary fields for Type II strings come from chiral and twisted-chiral operators in the Calabi-Yau CFT [23]. These operators are classified by their charges $\left(q_{L}, q_{R}\right)$ under $\left(\mathcal{J}_{L}, \mathcal{J}_{R}\right)$. Since they are (anti-)chiral their conformal weight is determined by their charge as $h=\frac{1}{2}|q|$. Operators of charge $(-1,-1)$ are annihilated by both $\left(\mathcal{G}_{L}^{-}, \mathcal{G}_{R}^{-}\right)$ and $\left(\Omega_{L}^{-}, \Omega_{R}^{-}\right)$and the ones with charge $(-1,1)$ are annihilated by $\left(\mathcal{G}_{L}^{-}, \mathcal{G}_{R}^{+}\right)$and $\left(\Omega_{L}^{-}, \Omega_{R}^{+}\right)$. 
Together with their complex conjugates, these operators describe the Kähler and complex compactification moduli respectively. Let $\Phi^{a}$ and $\Psi^{i}$ be the operators with charges $(-1,-1)$ and $(-1,1)$ respectively. Then the vertex operators are given by

$$
\Xi=M_{a} \Phi^{a}, \quad \Sigma=H_{i} \Psi^{i}
$$

where $a$ runs from 1 to $h^{1,1}$ (the number of Kähler parameters), $i$ runs from 1 to $h^{2,1}$ (the number of complex structure deformations), and $M_{a}$ and $H_{i}$ are space-time superfields. Since $\Xi$ and $\Sigma$ are charged, appropriate physical state conditions are

$$
\begin{aligned}
& \left(G_{L}^{+}\right)_{-\frac{1}{2}} \Xi=\left(G_{R}^{-}\right)_{-\frac{1}{2}} \Xi=\left(G_{L}^{-}\right)_{\frac{1}{2}} \Xi=\left(G_{R}^{+}\right)_{\frac{1}{2}} \Xi=0, \\
& \left(G_{L}^{+}\right)_{-\frac{1}{2}} \Sigma=\left(G_{R}^{+}\right)_{-\frac{1}{2}} \Sigma=\left(G_{L}^{-}\right)_{\frac{1}{2}} \Sigma=\left(G_{R}^{-}\right)_{\frac{1}{2}} \Sigma=0 .
\end{aligned}
$$

Using (2.7) and (2.8) these conditions for Type IIA imply that $M_{a}$ is chiral superfield and it is physical when $\nabla_{L}^{2} M_{a}=\nabla_{R}^{2} M_{a}=0$. It therefore describes an $N=2$ vector superfield. Similarly, $H_{i}$ is a twisted-chiral superfield which is physical when $\nabla_{L}^{2} H_{i}=$ $\bar{\nabla}_{R}^{2} H_{i}=0$, describing an $N=2$ tensor multiplet. In the case of Type IIB we use (2.9), and the roles of $M_{a}$ and $H_{i}$ are reversed. The form (2.14) is convenient for describing deformations of the hybrid string action. This is the usual description of the massless physical states of the theory. There are, however, alternative descriptions. We will discuss this for the Type IIA case only as it can be easily modified for Type IIB.

First we note that $\Xi$ and $\Sigma$ can also be described by uncharged operators. This comes from the fact that $e^{\rho_{L}} d_{L}^{2}$ and $e^{-\rho_{L}} \bar{d}_{L}^{2}$ together with their right moving (keeping in mind (2.8)) counterparts are invertable, that is

$$
\left(e^{\rho_{L}} d_{L}^{2}\right)_{-\frac{1}{2}}\left(e^{-\rho_{L}} \theta_{L}^{2}\right)=1, \quad\left(e^{-\rho_{L}} \bar{d}_{L}^{2}\right)_{-\frac{1}{2}}\left(e^{\rho_{L}} \bar{\theta}_{L}^{2}\right)=1
$$

Using left- and right-moving combinations of $e^{-\rho_{L}} \theta_{L}^{2}$ and $e^{\rho_{L}} \bar{\theta}_{L}^{2}$ it is therefore possible to write

$$
\begin{gathered}
\xi=\left(e^{\rho_{L}} \bar{\theta}_{L}^{2}\right)\left(e^{-\rho_{R}} \bar{\theta}_{R}^{2}\right) \Xi=\bar{\theta}_{L}^{2} \bar{\theta}_{R}^{2} M_{a} e^{\rho_{L}-\rho_{R}} \Phi^{a}, \\
\sigma=\left(e^{\rho_{L}} \bar{\theta}_{L}^{2}\right)\left(e^{\rho_{R}} \theta_{R}^{2}\right) \Sigma=\bar{\theta}_{L}^{2} \theta_{R}^{2} H_{i} e^{\rho_{L}+\rho_{R}} \Psi^{i} .
\end{gathered}
$$

Because $\theta$ appears explicitly in the equations above, $\xi$ and $\sigma$ do not look supersymmetric. Remembering, however, that $M_{a}$ and $H_{i}$ are chiral and twisted chiral allows us to write $M_{a}=\bar{\nabla}_{L}^{2} \bar{\nabla}_{R}^{2}\left(\bar{\theta}_{L}^{2} \bar{\theta}_{R}^{2} M_{a}\right)$ and $H_{i}=\bar{\nabla}_{L}^{2} \nabla_{R}^{2}\left(\bar{\theta}_{L}^{2} \theta_{R}^{2} H_{i}\right)$ or in a more general gauge, 
$M_{a}=\bar{\nabla}_{L}^{2} \bar{\nabla}_{R}^{2} m_{a}$ and $H_{i}=\bar{\nabla}_{L}^{2} \nabla_{R}^{2} h_{i}$, with complex unconstrained $m_{a}$ and $h_{i}$. The final result is that the uncharged operators can be written as

$$
\xi=m_{a} e^{\rho_{L}-\rho_{R}} \Phi^{a}, \quad \sigma=h_{i} e^{\rho_{L}+\rho_{R}} \Psi^{i}
$$

From this one can see that $m_{a}$ and $h_{i}$ play the role of prepotentials for $M_{a}$ and $H_{i}$, in analogy with the vertex operator for the supergravity sector. Using (2.7) and (2.8) one can show that these operators are uncharged with respect to the full superconformal algebra. The uncharged operators are essential for computing scattering amplitudes.

The extended worldsheet superconformal symmetry also allows different descriptions of vertex operators. Using the $s u(2)$ current algebra one can transform, for example, a chiral operator of the original superconformal algebra (2.7) to an anti-chiral field of (2.12). As will be shown below, it is useful to have operators with positive charge in the leftmoving sector and negative charge in the right-moving one. The vertex operators in (2.14) do not satisfy this requirement. Applying $J_{L}^{++}$on $\Xi$ we have

$$
\left(J_{L}^{++}\right)_{0}(\Xi)=M_{a} e^{-\rho_{L}} \Omega_{L}^{+}\left(\Phi^{a}\right)
$$

where $\Omega_{L}^{+}\left(\Phi^{a}\right)$ is twisted-chiral primary field in the Calabi-Yau CFT with charge $(2,-1)$ under $\left(\mathcal{J}_{L}, \mathcal{J}_{R}\right)$. The same procedure is applied to $\Sigma$ :

$$
\left(J_{L}^{++}\right)_{0}\left(J_{R}^{++}\right)_{0} \Sigma=H_{i} e^{-\rho_{L}-\rho_{R}} \Omega_{L}^{+}\left(\Omega_{R}^{-}\left(\Psi^{i}\right)\right)
$$

where $\Omega_{L}^{+}\left(\Omega_{R}^{-}\left(\Psi^{i}\right)\right)$ has charge $(2,-2)$. All operators generated from the actions of $\Omega_{L, M}^{ \pm}$ on the original $(-1,-1),(-1,1),(1,1)$ and $(1,-1)$ rings can be organized in four different Hodge diamonds. The one with the convenient charges is the one shown below:

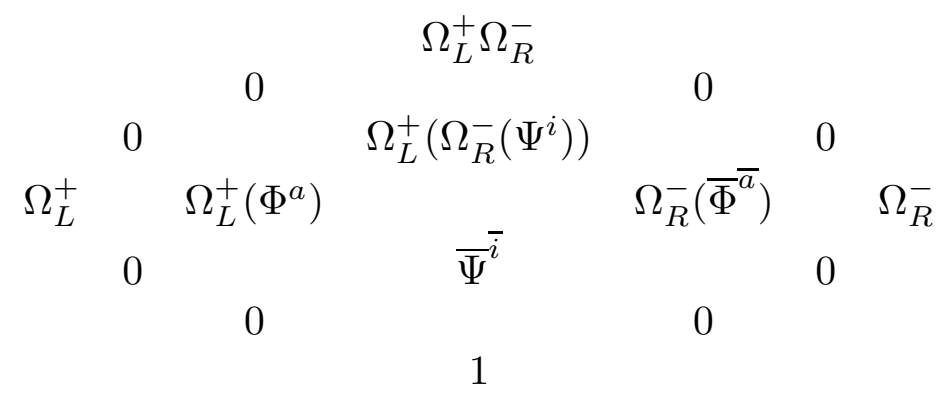

Table 1. The Hodge diamond of Calabi-Yau threefold CFT operators with positive left-moving charge and negative right-moving charge. 


\subsection{Deformations of the Action}

Deformations of the action, i.e. integrated vertex operators, should have conformal weight $(1,1)$ and preserve the $N=2$ superconformal algebra. Integrated vertex operators are also used to compute scattering amplitudes with more than three external states. Starting with $U$ the only operator that satisfies these conditions is

$$
\delta S_{U}=\int d^{2} z\left|G_{-\frac{1}{2}}^{+} G_{-\frac{1}{2}}^{-}\right|^{2} U
$$

where $|\cdot|^{2}$ means left- right-moving product. The explicit form of this vertex operator can be used to derive the full action in a general curved background [22]. Compactificationdependent states can also be used to deform the action. These are important for the description of general backgrounds with fluxes and warping. In the case of $M_{a}$, the vertex operator takes the form

$$
\begin{aligned}
\delta S_{M_{a}}= & \int d^{2} z\left[\left(G_{L}^{+}\right)_{-\frac{1}{2}}\left(G_{R}^{-}\right)_{-\frac{1}{2}} \Xi+c . c .\right]=\int d^{2} z\left[\left|G_{-\frac{1}{2}}^{+} G_{-\frac{1}{2}}^{-}\right|^{2} \xi+c . c .\right]= \\
& \int d^{2} z\left[M_{a} \mathcal{G}_{L}^{+}\left(\mathcal{G}_{R}^{+}\left(\Phi^{a}\right)\right)+e^{\rho_{L}-\rho_{R}} d_{L}^{\alpha} d_{R}^{\beta}\left(\nabla_{L \alpha} \nabla_{R \beta} M_{a}\right) \Phi^{a}+\right. \\
& \left.e^{\rho_{L}} d_{L}^{\alpha}\left(\nabla_{L \alpha} M_{a}\right) \mathcal{G}_{R}^{+}\left(\Phi^{a}\right)+e^{-\rho_{R}} d_{R}^{\alpha}\left(\nabla_{R \alpha} M_{a}\right) \mathcal{G}_{L}^{+}\left(\Phi^{a}\right)+c . c .\right],
\end{aligned}
$$

where $M_{a}=\bar{\nabla}_{R}^{2} \bar{\nabla}_{L}^{2} m_{a}$ is the chiral field strength. If $M_{a}$ is a constant superfield only the first term survives which corresponds to the usual result in the RNS formalism. The other terms are required in the supersymmetric formalism to ensure full superconformal invariance. Deformations corresponding to $H_{i}$ can be computed similarly:

$$
\begin{aligned}
\delta S_{H_{i}} & =\int d^{2} z\left[\left(G_{L}^{+}\right)_{-\frac{1}{2}}\left(G_{R}^{+}\right)_{-\frac{1}{2}} \Sigma+c . c .\right]=\int d^{2} z\left[\left|G_{-\frac{1}{2}}^{+} G_{-\frac{1}{2}}^{-}\right|^{2} \sigma+c . c .\right] \\
& =\int d^{2} z\left[H_{i} \mathcal{G}_{L}^{+}\left(\mathcal{G}_{R}^{-}\left(\Psi^{i}\right)\right)+e^{\rho_{L}+\rho_{R}} d_{L}^{\alpha} \bar{d}_{R}^{\dot{\beta}}\left(\nabla_{L \alpha} \bar{\nabla}_{R \dot{\beta}} H_{i}\right) \Psi^{i}\right. \\
& \left.+e^{\rho_{L}} d_{L}^{\alpha}\left(\nabla_{L \alpha} H_{i}\right) \mathcal{G}_{R}^{-}\left(\Psi^{i}\right)+e^{\rho_{R}} \bar{d}_{R}^{\dot{\alpha}}\left(\bar{\nabla}_{R \dot{\alpha}} H_{i}\right) \mathcal{G}_{L}^{+}\left(\Psi^{i}\right)+c . c .\right]
\end{aligned}
$$




\subsection{Supersymmetric Amplitudes}

Supersymmetric amplitudes can be computed in a straightforward way using the rules of the hybrid formalism [24,25] in four dimensions. Amplitudes are calculated by twisting the superconformal algebra. This has the effect of shifting the conformal weights $\left(h_{L}, h_{R}\right) \rightarrow\left(h_{L}-\frac{q_{L}}{2}, h_{R}-\frac{q_{R}}{2}\right)$ so that all operators defined in the zero-mode measure have conformal weight zero, as they should. The twisting is also responsible for a charge anomaly of 2 in the left- and right-moving sector, which is cancelled by the measure. Due to the charge anomaly in the algebra of (2.7), we will have to use all of the operators defined above to obtain non-vanishing amplitudes. The first step is to define the measure over zero-modes. To this end it should be observed that the integral of the product of the holomorphic and anti-holomorphic forms over the Calabi-Yau is proportional to the volume

$$
i \int_{C Y_{3}} \Omega \wedge \bar{\Omega}=\frac{4}{3} \operatorname{Vol}\left(\mathrm{CY}_{3}\right) .
$$

As was shown above, these forms are represented by $\Omega_{L}^{+}$and $\Omega_{R}^{-}$. In the internal CFT we therefore define

$$
\left\langle\Omega_{L}^{+} \Omega_{R}^{-}\right\rangle_{\mathrm{CY}_{3}}=1
$$

In the non-compact sector momentum conservation is ensured by integrating over space-time. In addition, we have to remove the zero-modes of the fermionic coordinates $\theta$; their conjugate momenta $p$ have no zero modes on the sphere. The final ingredient is the measure for the chiral bosons $\left(\rho_{L}, \rho_{R}\right)$. The final form of measure is

$$
\left\langle\theta_{R}^{2} \bar{\theta}_{L}^{2} \theta_{R}^{2} \bar{\theta}_{R}^{2} e^{-\rho_{L}-\rho_{L}} \Omega_{L}^{+} \Omega_{R}^{-}\right\rangle=1
$$

The first non-vanishing amplitude is the three point function and due to $\operatorname{SL}(2, \mathbb{R})$ invariance, the three vertex operators should be unintegrated. The charge anomaly in the $N=2$ twisted algebra factorizes between space-time and Calabi-Yau sectors; a -1 contribution should come from the chiral bosons and +3 from the (anti-)holomorphic forms. This requirement narrows down the possible choices.

As a first example, let us compute the three-point function in the Type IIA string for the $\bar{H}_{\bar{i}}$ moduli. Two charged $\bar{\Sigma}$ and one uncharged vertex operator $\bar{\sigma}=\bar{h}_{\bar{i}} e^{-\rho_{L}-\rho_{R}} \bar{\Psi}^{\bar{i}}$ will

8 Note that the measure is of D-term type. Since some superconformal generators have trivial cohomology it is also possible to write chiral and twisted-chiral F-term measures [9, 10]. 
be needed. The chiral ring structure of operators in Table 1 is essential in the computation. It is not hard to see that the correlation between three $\bar{\Psi}_{\mathrm{S}}$ is [23]

$$
\bar{\Psi}^{\bar{i}} \times \bar{\Psi}^{\bar{j}} \times \bar{\Psi}^{\bar{k}}=\Omega_{L}^{+} \Omega_{R}^{-} \mathcal{C}^{\overline{i j k}}
$$

where $\mathcal{C}^{\overline{i j k}}$ are the $h^{2,1}$ intersection numbers. The final answer is

$$
\left\langle\bar{\Sigma}_{1} \bar{\Sigma}_{2} \bar{\sigma}_{3}\right\rangle=\int d^{4} x d^{2} \theta_{L} d^{2} \bar{\theta}_{L} d^{2} \theta_{R} d^{2} \bar{\theta}_{R} \bar{H} \overline{\bar{H}} \overline{\bar{j}} \overline{\bar{k}}_{\bar{k}} \mathcal{C}^{\overline{i j k}}
$$

where the integration over $\theta$ comes from the zero-mode measure. Since $\bar{H}$ 's are twistedchiral and $\bar{h}_{\bar{k}}$ is unconstrained, we can further perform integration over $d^{2} \theta_{L} d^{2} \bar{\theta}_{R}$ to get

$$
\left\langle\bar{\Sigma}_{1} \bar{\Sigma}_{2} \bar{\sigma}_{3}\right\rangle=\int d^{4} x d^{2} \bar{\theta}_{L} d^{2} \theta_{R} \bar{H} \bar{i} \bar{j} \bar{H} \bar{C} \mathcal{C}^{\overline{i j k}}
$$

which is the expected result. Similarly, it is possible to compute the amplitude for $H_{i}$ using two $\left(J_{L}^{++}\right)_{0}\left(J_{R}^{++}\right)_{0} \Sigma=H_{i} e^{-\rho_{L}-\rho_{R}} \Omega_{L}^{+}\left(\Omega_{R}^{-}\left(\Psi^{i}\right)\right)$ vertex operators and one $\sigma$. The calculation is slightly more involved due to the correlation between the chiral bosons and $\mathrm{CY}_{3}$ operators. A shorter path, which gives the same answer, is to just take the complex conjugate of the previous amplitude

$$
\left\langle\left[\left(J_{L}^{++}\right)_{0}\left(J_{R}^{++}\right)_{0} \Sigma_{1}\right]\left[\left(J_{L}^{++}\right)_{0}\left(J_{R}^{++}\right)_{0} \Sigma_{2}\right] \sigma_{3}\right\rangle=\int d^{4} x d^{2} \theta_{L} d^{2} \bar{\theta}_{R} H_{i} H_{j} H_{k} \mathcal{C}^{i j k}
$$

Now let us compute amplitudes involving $M_{a}$. We need two operators of the type $\left(J_{L}^{++}\right)_{0}(\Xi)=M_{a} e^{-\rho_{L}} \Omega_{L}^{+}\left(\Phi^{a}\right)$ and one $\xi$. This time we have

$$
e^{-\rho_{L}} \Omega_{L}^{+}\left(\Phi^{a}\right) \times e^{-\rho_{L}} \Omega_{L}^{+}\left(\Phi^{b}\right) \times e^{\rho_{L}-\rho_{R}} \Phi^{c}=e^{-\rho_{L}-\rho_{R}} \Omega_{L}^{+} \Omega_{R}^{-} \mathcal{K}^{a b c},
$$

where $\mathcal{K}^{a b c}$ are $h^{1,1}$ intersection numbers. Note that the factors of $e^{\rho_{L}}$ and $e^{-\rho_{L}}$ are needed to remove poles and zeros in the correlators of operators in the Hodge diamond. The amplitude becomes

$$
\left\langle\left[\left(J_{L}^{++}\right)_{0} \Xi_{1}\right]\left[\left(J_{L}^{++}\right)_{0} \Xi_{2}\right] \xi_{3}\right\rangle=\int d^{4} x d^{2} \theta_{L} d^{2} \theta_{R} M_{a} M_{b} M_{c} \mathcal{K}^{a b c}
$$

The analogous formula for anti-chiral fields is

$$
\left\langle\left[\left(J_{R}^{++}\right)_{0} \bar{\Xi}_{1}\right]\left[\left(J_{R}^{++}\right)_{0} \bar{\Xi}_{2}\right] \bar{\xi}_{3}\right\rangle=\int d^{4} x d^{2} \bar{\theta}_{L} d^{2} \bar{\theta}_{R} \bar{M}_{\bar{a}} \bar{M}_{\bar{b}} \bar{M}_{\bar{c}} \overline{\mathcal{K}}^{\bar{a} \bar{b} \bar{c}}
$$


All other three-point amplitudes involving only moduli states are zero. We now turn to amplitudes with more than three points. These amplitudes have the general form

$$
\left\langle\mathcal{V}_{1} \mathcal{V}_{2} v_{3} \prod_{i=4}^{n} \int d^{2} z_{i} \mathcal{U}_{i}\right\rangle
$$

where $\mathcal{V}$ are charged, unintegrated vertex operators, $v$ is uncharged and the $\mathcal{U}_{i}$ are integrated vertex operators like (2.20). All the operators used in the previous computations are the only ones with zero conformal weight in the twisted theory. Furthermore, they saturate the charge anomaly in the correlation functions. These two facts imply that if we consider higher point amplitudes, only terms like the first one in (2.20) are going to enter the computation, so in the hybrid formalism the usual non-renormalization theorems [23] for tree level amplitudes apply. The charge anomaly will also be useful in Section 4, when we compute amplitudes involving Ramond-Ramond flux. Four-point amplitudes can be calculated similarly, and give the special geometry equations relating the curvature tensor to the metric and Yukawa couplings (see e.g. the second reference in [23]). Since we are not going to use this type of amplitude, we will not discuss it further. The problem of computing one loop amplitudes as in reference [25] for compactification-dependent states is still open.

\section{Dimensional Reduction, Quotients and $G_{2}$ Holonomy}

In this section we discuss all multiplets defined above and introduce the superspace reduction that relates them to theories with less supersymmetry. We then turn to the description of the Type IIA string on a $\left(\mathrm{CY}_{3} \times S^{1}\right) / \mathbb{Z}_{2}$ quotient with $G_{2}$ holonomy.

\subsection{The Superspace ${ }^{N} d={ }^{2} 4$ and Its Reductions}

The ${ }^{N} d={ }^{2} 4$ chiral $M$ and twisted-chiral $H$ field strengths are defined by the constraints

$$
0=\bar{\nabla}_{L \dot{\alpha}} M=\bar{\nabla}_{R \dot{\alpha}} M \quad, \quad 0=\bar{\nabla}_{L \dot{\alpha}} H=\nabla_{R \alpha} H
$$

implying that they can be written in terms of unconstrained complex prepotentials $m$ and $h$

$$
M=\bar{\nabla}_{L}^{2} \bar{\nabla}_{R}^{2} m \quad, \quad H=\bar{\nabla}_{L}^{2} \nabla_{R}^{2} h
$$


In addition, the reality conditions

$$
0=\nabla_{L}^{2} M-\bar{\nabla}_{R}^{2} \bar{M} \quad, \quad 0=\nabla_{L}^{2} H-\nabla_{R}^{2} \bar{H}
$$

are imposed, resulting in the component expressions

$$
\begin{gathered}
M=\varphi+\theta_{L}^{\alpha} \psi_{\alpha}+\theta_{R}^{\alpha} \lambda_{\alpha}+\theta_{L}^{2} F+\theta_{R}^{2} \bar{F}+\theta_{L}^{\alpha} \theta_{R}^{\beta}\left(\varepsilon_{\alpha \beta} D+f_{\alpha \beta}\right)+\ldots \\
H=\ell+\theta_{L}^{\alpha} \eta_{\alpha}+\bar{\theta}_{R}^{\dot{\alpha}} \bar{\xi}_{\dot{\alpha}}+\theta_{L}^{2} y+\bar{\theta}_{R}^{2} \bar{y}+\theta_{L}^{\alpha} \bar{\theta}_{R}^{\dot{\alpha}}\left(i \partial_{\alpha \dot{\alpha}} l+\tilde{H}_{\alpha \dot{\alpha}}\right)+\ldots
\end{gathered}
$$

where the ellipses denote auxiliary terms. The reality conditions are necessary to ensure that $F_{m n}=\left(\gamma_{m n}\right)^{\alpha \beta} f_{\alpha \beta}+$ h.c. and $\tilde{H}_{m}=\epsilon_{m n p q} H^{n p q}$ satisfy the appropriate Bianchi identities. They also put the theory partially on-shell, an inevitability of non-harmonic superspaces. Fortunately, this shortcoming will not hamper our analysis too much allowing us to avoid introducing harmonic superspaces in this work.

In what follows, we will make use of the dimensional reduction of this superspace from ${ }^{N} d={ }^{2} 4$ to ${ }^{N} d={ }^{4} 3$. We choose to single out $y=x_{2}$ for this purpose. We then use $i\left(\sigma^{2}\right)_{\alpha \dot{\alpha}}$ to convert all dotted spinor indices to undotted ones and define $\left(\gamma^{m}\right)_{\alpha}{ }^{\beta}=i\left(\sigma^{2} \sigma^{m}\right)_{\alpha}{ }^{\beta}$ to be the real three-dimensional Dirac matrices. These matrices are symmetric upon lowering an index. The superspace coordinates can now be taken to be $\left(x^{m}, y, \theta_{L}^{\alpha}, \bar{\theta}_{L}^{\alpha}, \theta_{R}^{\alpha}, \bar{\theta}_{R}^{\alpha}\right)$.

$$
\begin{gathered}
\left\{\nabla_{L \alpha}, \nabla_{L \beta}\right\}=0 \quad, \quad\left\{\nabla_{R \alpha}, \nabla_{R \beta}\right\}=0 \\
\left\{\bar{\nabla}_{L \alpha}, \bar{\nabla}_{L \beta}\right\}=0 \quad, \quad\left\{\bar{\nabla}_{R \alpha}, \bar{\nabla}_{R \beta}\right\}=0 \\
\left\{\nabla_{L \alpha}, \bar{\nabla}_{L \beta}\right\}=-2 i \partial_{\alpha \beta}-2 \varepsilon_{\alpha \beta} \partial_{y} \quad, \quad\left\{\nabla_{R \alpha}, \bar{\nabla}_{R \beta}\right\}=-2 i \partial_{\alpha \beta}-2 \varepsilon_{\alpha \beta} \partial_{y}
\end{gathered}
$$

A new feature of ${ }^{N} d={ }^{4} 3$ superspace is the involution exchanging $\theta_{L}^{\alpha} \leftrightarrow \bar{\theta}_{L}^{\alpha}, \theta_{R}^{\alpha} \leftrightarrow \bar{\theta}_{R}^{\alpha}$, and taking $y \mapsto-y$. 9 More useful for our purposes is the combination of this involution with the usual hermitian conjugation (denoted ${ }^{-}$) which we will denote by $\circ$. Note that although $\circ$ involves the whole superspace, effectively it only acts on components of (twisted)chiral superfields since the two involutions of which it is composed together fix $\theta_{L, R}$ and $\bar{\theta}_{L, R}$.

9 Starting with Type I superspace in ten dimensions and working down, one finds that it is not possible to define an involution of this type preserving more than an $\mathrm{SO}(1,5)$ Lorentz symmetry. This corresponds to compactifification on a $K 3$ surface and is a simple way to see why the first special holonomy manifold occurs in four dimensions. 
Having identified a new involution it is natural to consider its eigenspaces. We therefore introduce new projection operators $\operatorname{Re}_{\circ}=\frac{1}{2}(1+\circ)$ and $\operatorname{Im}_{\circ}=\frac{1}{2 i}(1-\circ)$ acting on superfields. Similarly to the ordinary real and imaginary subspaces of complexified superspace, the o-real and o-imaginary superspaces are half-supersymmetric. In this way, the ${ }^{N} d={ }^{4} 3$ representations are reduced to ${ }^{N} d={ }^{2} 3$.

Let let $X=\operatorname{Re}_{\circ} N$ and $Y=\operatorname{Im}_{\circ} N$ denote the o-real and o-imaginary parts of a general ${ }^{N} d={ }^{2} 4$ superfield $N$. Then $N=X+i Y$. Note that $\bar{X} \neq X$ and similarly for $Y$ so these fields are not real with respect to the original ${ }^{-}$-conjugation. However, it is easy to see that under left-moving supersymmetry transformations $\delta_{L} N=\left(\epsilon_{L}^{\alpha} Q_{L \alpha}+\bar{\epsilon}_{L}^{\alpha} \bar{Q}_{L \alpha}\right) N$,

$$
\begin{aligned}
& \delta_{L} X=(\epsilon+\bar{\epsilon})_{L}^{\alpha}(Q+\bar{Q})_{L \alpha} X-(\epsilon-\bar{\epsilon})_{L}^{\alpha}(Q-\bar{Q})_{L \alpha} Y \\
& \delta_{L} Y=(\epsilon+\bar{\epsilon})_{L}^{\alpha}(Q+\bar{Q})_{L \alpha} Y+(\epsilon-\bar{\epsilon})_{L}^{\alpha}(Q-\bar{Q})_{L \alpha} X
\end{aligned}
$$

and similarly for the right-moving supersymmetries. The combination $(\epsilon+\bar{\epsilon})_{L}$ parameterizes a supersymmetry which is realized linearly on $X$ and $Y$ separately while $(\epsilon-\bar{\epsilon})_{L}$ mixes the two. Therefore, $X$ and $Y$ are ${ }^{N} d={ }^{2} 3$ superfields. We will henceforth use $X$ and $Y$ to denote the o-real and o-imaginary parts of a chiral superfield $N=M$. Similarly $S=\operatorname{Re}_{\circ} H$ and $T=\operatorname{Im}_{\circ} H$ will denote the half-supersymmetric projections of the twisted-chiral superfield $H$.

It is easy to show by covariant projection that the ${ }^{N} d={ }^{2} 3$ superfields $X, S$ and $Y, T$ are strengths for a (partially on-shell) vector multiplet and a scalar multiplet respectively. For example for the field strength components of $X$ and $Y$ we find

$$
\begin{gathered}
\nabla_{L \alpha} \nabla_{R \beta} X \mid=\left(\gamma^{m n}\right)_{\alpha \beta} F_{m n} \\
\nabla_{L \alpha} \nabla_{R \beta} Y \mid=\left(\gamma^{m}\right)_{\alpha \beta}\left(\partial_{y} A_{m}-\partial_{m} a\right)-i \varepsilon_{\alpha \beta} D \\
\nabla_{L \alpha} \bar{\nabla}_{R \beta} S \mid=\left(\gamma^{m}\right)_{\alpha \beta} \tilde{H}_{m}-\varepsilon_{\alpha \beta} \partial_{y} l \\
\nabla_{L \alpha} \bar{\nabla}_{R \beta} T \mid=\left(\gamma^{m}\right)_{\alpha \beta} \partial_{m} l+\varepsilon_{\alpha \beta} \tilde{H}_{y}
\end{gathered}
$$

where we have retained the $\partial_{y}$-terms for use in section 4.2 where we will interpret them as fluxes coupling to space-time defects.10

10 If a higher-dimensional theory is written in a lower-dimensional superspace notation then it is often the case that the 'scalar' field strengths in the extra directions are related by equations of motion to $F$ - and $D$-terms. For example, in six-dimensional SYM the $D$-term of the gauge field $V$ is related to the flux in the 5 - and 6 -directions by the equation of motion $D=F_{56}$ [26, 11] It is therefore possible to turn on these particular fluxes without breaking supersymmetry if we simultaneously give vevs of the same magnitude to these auxiliary terms. 
In the basis defined by $\theta_{\alpha}^{+}=\left(\theta_{L \alpha}+\theta_{R \alpha}\right)$ and $\theta_{\alpha}^{-}=\left(\theta_{L \alpha}-\theta_{R \alpha}\right), N=1$ decompositions may be represented (up to certain auxiliary fields) as $X=\Phi\left(\theta^{+}\right)+\theta^{-\alpha} W_{\alpha}\left(\theta^{+}\right.$) where $\Phi$ and $W_{\alpha}$ are the standard ${ }^{N} d={ }^{1} 3$ field strengths for a scalar and vector multiplet while $Y=\Phi^{+}\left(\theta^{+}\right)+\Phi^{-}\left(\theta^{-}\right)$is the direct sum of two scalar superfields. The analogous statements hold for $S$ and $T$ in the basis $\theta_{\alpha}^{+}=\left(\theta_{L \alpha}+\bar{\theta}_{R \alpha}\right), \theta_{\alpha}^{-}=\left(\theta_{L \alpha}-\bar{\theta}_{L \alpha}\right)$. In this case, the field strength $\Upsilon_{\alpha}$ analogous to $W_{\alpha}$ is the $d=3$ version of a variant representation of the tensor multiplet.

Finally, let us comment very briefly on the structure of a real scalar prepotential $U$. As mentioned in section 2.3, the gravitational multiplet is represented such a field. As described in detail in reference [22], it has a gauge invariance $\delta U=\nabla_{L}^{2} \Lambda_{L}+\bar{\nabla}_{L}^{2} \bar{\Lambda}_{L}+$ $\nabla_{R}^{2} \Lambda_{R}+\bar{\nabla}_{R}^{2} \bar{\Lambda}_{R}$ and can be put into the Wess-Zumino gauge

$$
U=\left(h_{m n}+b_{m n}+l^{+-} \eta_{m n}\right) \sigma_{\alpha \dot{\alpha}}^{m} \sigma_{\beta \dot{\beta}}^{n} \theta_{L}^{\alpha} \bar{\theta}_{L}^{\dot{\alpha}} \theta_{R}^{\beta} \bar{\theta}_{R}^{\dot{\beta}}+\ldots
$$

where the ellipsis denotes higher-dimensional fields which will not enter our considerations. Upon reduction under $\circ$, it is easy to check that $\operatorname{Re}_{\circ} U$ contains $h_{m n}, b_{m n}$, and $h_{y y}$ and $\operatorname{Im}_{\circ} U$ contains $h_{m y}, b_{m y}$, and $l^{+-}$. This result will be important when we discuss warping in section 4 .

\section{2. $G_{2}$ Structure and $\mathbb{Z}_{2}$ Quotient}

The formalism developed in section 2 has $N=4$ supersymmetry in three dimensions. There are many ways to obtain a theory with a smaller amount of supersymmetry. Given an initial setup in which the compactification manifold is of the form $\mathrm{CY}_{3} \times S^{1}$, the obvious way to break half of the supersymmetry is by a $\mathbb{Z}_{2}$ quotient, which is a well-known way to obtain $G_{2}$ holonomy manifolds. The resulting spectrum is equivalent to a direct type IIA reduction on a general $G_{2}$ holonomy manifold. This example breaks supersymmetry within left- and right-moving sectors as we discuss presently. A second way to reduce the amount of supersymmetry breaks between the two sectors by the introduction of some flux. We demonstrate the effects of such background fluxes in four dimensions in section 4 by explicit worldsheet computations.

Our starting point is a Calabi-Yau 3-fold $\mathrm{CY}_{3}$ with complex structure $J$, symplectic (1,1)-form $\omega$, and holomorphic volume (3,0)-form $\Omega$. The Hodge structure is the familiar one with variable $h^{1,1}$ and $h^{2,1}$. Consider a conjugation acting freely on the Calabi-Yau defined such that $J \rightarrow-J, \omega \rightarrow-\omega$, and $\Omega \rightarrow \bar{\Omega}$. We extend this action to the circle with 
coordinate $y \in(-\pi, \pi]$ by the reflection $y \mapsto-y$. Let us denote the combined operation by $\sigma: \mathrm{CY}_{3} \times S^{1} \rightarrow \mathrm{CY}_{3} \times S^{1}$. Although $\sigma$ fixes $y=0$ and $y=\pi$ on $S^{1}$, its action on $\mathrm{CY}_{3}$ is free and therefore the quotient $X=\left(\mathrm{CY}_{3} \times S^{1}\right) / \sigma$ is smooth. The 3-form $\Phi=\omega \wedge \mathrm{d} y+\operatorname{Re} \Omega$, being invariant under the action of $\sigma$, descends to $X$ providing it with a $G_{2}$-structure [27].

Under the action of $\sigma$ the cohomology of the Calabi-Yau descends to the following. Since the space of 2-forms is real, it splits as $H^{1,1}=H_{+}^{1,1} \oplus H_{-}^{1,1}$ where the $H_{ \pm}^{1,1}$ eigenspaces have the indicated eigenvalues. The odd forms are reflected through the vertical of the Hodge diamond since the involution acts as a conjugation. Therefore, the eigenspaces are subspaces of the sums $H^{3,0} \oplus H^{0,3}$, spanned by $\operatorname{Re} \Omega$ and $\operatorname{Im} \Omega$, and $H^{2,1} \oplus H^{1,2}$. Quotienting by $\sigma$ projects out the odd eigenspaces. The resulting cohomology of $X$ is real and has Betty numbers $b^{2}=h_{+}^{1,1}$ and $b^{3}=h_{-}^{1,1}+h^{2,1}+1$ where the $h_{-}^{1,1}$ terms in $b^{3}$ come from wedging with the 1-form $\mathrm{d} y$ on the circle.

We would like to extend this conjugation to the full superspace. Such an extension must be symmetric in its action on left-moving and right-moving fermionic coordinates. From the discussion in section 3, the obvious candidate is the o-involution. Assuming this, we are in position to determine the spectrum. We take for definiteness type IIA on the Calabi-Yau 3-fold. Then, as explained in section 2, the $h^{1,1}$ Kähler moduli are parameterized by the scalars in the ${ }^{N} d={ }^{2} 4$ vector multiplets $M_{a}$ while the $h^{2,1}$ complex moduli are embedded in the hypermultiplets $H_{i}$. Let us write the relevant vertex operators as

$$
\sum_{a=1}^{h^{1,1}} M_{a} \Phi^{a}+\sum_{i=1}^{h^{2,1}} H_{i} \Psi^{i}+\text { h.c. }
$$

where the $\Phi^{a}$ generate $H^{1,1}$ and the $\Psi^{i}$ generate $H^{2,1}$. From the discussion above, we see that this expression decomposes under the extended conjugation as

$$
\sum_{a=1}^{h_{+}^{1,1}} X_{a} \Phi_{+}^{a}+\sum_{a=1}^{h_{-}^{1,1}} Y_{a} \Phi_{-}^{a}+\sum_{i=1}^{h^{2,1}} S_{i} \operatorname{Re} \Psi^{i}+\text { h.c. }
$$

This result agrees with the standard component analysis [28,20]. With this construction on can take a solvable model for the quotient $X=\left(\mathrm{CY}_{3} \times S^{1}\right) / \sigma$ such as [29] and use the hybrid formalism to compute supersymmetric amplitudes. 


\section{Internal Ramond-Ramond Fluxes and Deformations}

We will now apply the methods of the hybrid formalism to the problem of internal Ramond-Ramond fluxes. Computations of superpotentials can be done by calculating scattering amplitudes with appropriate operators. In [18] Lawrence and McGreevy have given a detailed discussion of the role of auxiliary fields and their meaning in terms of RR fluxes. We will use that analysis combined with the hybrid formalism to compute superpotentials and effects due to torsion and warping.

It has been known since the beginning of the study of supersymmetric theories that giving vevs to auxiliary fields can be used to break supersymmetry. The connection between auxiliary fields and internal RR fluxes was made in [30,16].

If $M_{a}\left(x, \theta_{L}, \theta_{R}\right)$ is a constant chiral superfield and there are no fermionic background or Lorentz breaking terms, we have the simple form

$$
M_{a}\left(\theta_{L}, \theta_{R}\right)=\phi_{a}+\theta_{L}^{2} F_{a}+\theta_{R}^{2} \bar{F}_{a}+\theta_{L}^{\alpha} \theta_{R}^{\beta} \epsilon_{\alpha \beta} D_{a},
$$

where $\left(F_{a}, \bar{F}_{a}, D_{a}\right)$ are auxiliary fields representing a combination of RR and NSNS fluxes [18]. For general values of $(F, \bar{F}, D)$ supersymmetry is completely broken. There are two general cases preserving half of the supersymmetry. In the first one a combination of $\left(\theta_{L}, \theta_{R}\right)$ is preserved $\theta_{L}+e^{i \gamma} \theta_{R}$ where $\gamma$ is a phase depending on the specific model of supersymmetry breaking. In this case $M_{a}$ has the form

$$
M_{a}=\phi_{a}+\left(\theta_{L}+e^{i \gamma} \theta_{R}\right)^{2} F_{a} .
$$

In the second case the right- (or left-)moving supercharges are broken, preserving the other. Then, $M_{a}$ takes the form

$$
M_{a}=\phi_{a}+\theta_{R}^{2} \bar{F}_{a},
$$

where $F_{a}$ is due to NS flux. In the case of $H_{i}$, the only combinations involving left- and right-moving product of $\theta$ are vectors in space-time which means that $H_{i}$ can only be used to describe NSNS fluxes.

$$
H_{i}=\psi_{i}+\theta_{L}^{2} y_{i},
$$

where the left moving supersymmetry is broken. The physical meaning of all these auxiliary fields depends on the choice of type IIA or type IIB string as discussed in detail in [18].

It was pointed out in [17, 18] that giving vevs to the auxiliary fields in compactification multiplets violates the physical state conditions (2.15) and (2.16) and that a possible 
solution to this apparent inconsistency is that the physical state conditions are modified when this type of flux is turned on. This means that giving vevs to those fields breaks $N=2$ superconformal invariance on the worldsheet. Instead of modifying the physical state condition we modify the physical state itself by observing that these deformations cannot be turned on alone. That is, there should be another deformation in the action that compensates the breaking due to non-zero values in the auxiliary components of $\Xi$ and $\Sigma$.

Let us analyze one specific case to be more explicit. Suppose we want to add a deformation of the action corresponding to $\delta \Xi=\left(\theta_{L}-\theta_{R}\right)^{2} F_{a} \Phi_{a}$, breaking a combination of left-right moving supersymmetry. This vertex operator does not satisfy the physical state condition (2.15). A clear way to see this in terms of the familiar language of chiral states is that the vertex operator $\left(\theta_{L}-\theta_{R}\right)^{2} F_{a} e^{-\rho_{L}+\rho_{R}} \Omega_{L}^{+}\left(\Omega_{R}^{+}\left(\Phi^{a}\right)\right)$ has a single pole with $G_{L}^{+}$and $G_{R}^{-}$viz.

$$
\begin{gathered}
G_{L}^{+}\left[\left(\theta_{L}-\theta_{R}\right)^{2} F_{a} e^{-\rho_{L}+\rho_{R}} \Omega_{L}^{+}\left(\Omega_{R}^{+}\left(\Phi^{a}\right)\right)\right] \rightarrow \frac{1}{z} e^{\rho_{R}} F_{a} \Omega_{L}^{+}\left(\Omega_{R}^{+}\left(\Phi^{a}\right)\right), \\
G_{R}^{-}\left[\left(\theta_{L}-\theta_{R}\right)^{2} F_{a} e^{-\rho_{L}+\rho_{R}} \Omega_{L}^{+}\left(\Omega_{R}^{+}\left(\Phi^{a}\right)\right)\right] \rightarrow \frac{1}{\bar{z}} e^{-\rho_{L}} F_{a} \Omega_{L}^{+}\left(\Omega_{R}^{+}\left(\Phi^{a}\right)\right),
\end{gathered}
$$

so it fails to be (anti-)chiral. (Note that $\mathcal{G}_{L}^{+}\left[\Omega_{L}^{+}\left(\Omega_{R}^{+}\left(\Phi^{a}\right)\right)\right] \rightarrow 0$ and $\mathcal{G}_{R}^{+}\left[\Omega_{L}^{+}\left(\Omega_{R}^{+}\left(\Phi^{a}\right)\right)\right] \rightarrow 0$.) To remedy this, one has to remember that one of the effects of fluxes is to generate torsions [19] and it turns out that at linearized level in the deformation semi-chiral and non-chiral (depending on the type of flux) operators have to be included in the internal CFT. These new operators are not physical by themselves either; only the combination is a consistent deformation of the background. This is the worldsheet counterpart to the target space result that torsions modify the closure conditions on the forms. A consequence of this in the hybrid formalism is that the theory does not factorize into two independent CFTs; space-time and internal superconformal generators are not conserved separately. Of course, the introduction of fluxes does not add new states in the spectrum implying that these new semi-chiral and non-chiral vertex operators should not be independent. The physical operator will have the form

$$
e^{-\rho_{L}+\rho_{R}} \Omega_{L}^{+}\left(\Omega_{R}^{+}(\delta \Xi)\right)+e^{\rho_{R}} W_{1}+e^{-\rho_{L}} W_{2},
$$

where $e^{\rho_{R}} W_{1}+e^{-\rho_{L}} W_{2}$ is a vertex operator representing the effect of torsion with charge $(1,-1)$ and we are assuming it does not depend on space-time and $\theta$. The (anti-)chirality conditions are now

$$
0=G_{L}^{+}\left[\left(\theta_{L}-\theta_{R}\right)^{2} F_{a} e^{-\rho_{L}+\rho_{R}} \Omega_{L}^{+}\left(\Omega_{R}^{+}\left(\Phi^{a}\right)\right)+e^{\rho_{R}} W_{1}+e^{-\rho_{L}} W_{2}\right]
$$




$$
\begin{aligned}
& \rightarrow \frac{1}{z}\left[F_{a} e^{\rho_{R}} \Omega_{L}^{+}\left(\Omega_{R}^{+}\left(\Phi^{a}\right)\right)+e^{\rho_{R}} \mathcal{G}_{L}^{+}\left(W_{1}\right)+e^{-\rho_{L}} \mathcal{G}_{L}^{+}\left(W_{2}\right)\right] \\
0 & G_{R}^{-}\left[\left(\theta_{L}-\theta_{R}\right)^{2} F_{a} e^{-\rho_{L}+\rho_{R}} \Omega_{L}^{+}\left(\Omega_{R}^{+}\left(\Phi^{a}\right)\right)+e^{\rho_{R}} W_{1}+e^{-\rho_{L}} W_{2}\right] \\
\rightarrow & \frac{1}{z}\left[F_{a} e^{-\rho_{L}} \Omega_{L}^{+}\left(\Omega_{R}^{+}\left(\Phi^{a}\right)\right)+e^{-\rho_{L}} \mathcal{G}_{R}^{+}\left(W_{2}\right)+e^{+\rho_{R}} \mathcal{G}_{R}^{+}\left(W_{1}\right)\right]
\end{aligned}
$$

whence we obtain four equations determining $W_{1}$ and $W_{2}$ :

$$
\begin{array}{ll}
F_{a} \Omega_{L}^{+}\left(\Omega_{R}^{+}\left(\Phi^{a}\right)\right)=-\mathcal{G}_{L}^{+}\left(W_{1}\right), & \mathcal{G}_{R}^{+}\left(W_{1}\right)=0, \\
F_{a} \Omega_{L}^{+}\left(\Omega_{R}^{+}\left(\Phi^{a}\right)\right)=-\mathcal{G}_{R}^{+}\left(W_{2}\right), & \mathcal{G}_{L}^{+}\left(W_{2}\right)=0 .
\end{array}
$$

In the large radius limit $\mathcal{G}_{L}^{+}$acts as $d y^{I} \partial_{I}$ and $\mathcal{G}_{R}^{+}$acts as $d \bar{y}^{\bar{I}} \partial_{\bar{I}}$ in our notation and the above equations are recognizable as the equations relating components of the intrinsic torsion to the un-deformed forms in the Calabi-Yau [19]. It should be stressed that we are considering only the first order in $F_{a}$. In the case of $\delta \Xi,\left(W_{1}, W_{2}\right)$ is a pair of semi-chiral and semi-anti-chiral vertex operators. If (4.2) is used instead to deform the action, only $W_{2}$ would be needed and the deformed compactification manifold will not be complex. Since $\left(W_{1}, W_{2}\right)$ are not chiral primaries, it not clear how to construct them in terms of operators corresponding to geometric objects. Nevertheless, at least in the classical limit, it should be possible to write a $\sigma$-model action including all possible corrections in $F_{a}$. This gives an exact form for $G_{L, R}^{ \pm}$and hence exact equations analogous to (4.4) and (4.5) for $\left(W_{1}, W_{2}\right)$.

It is interesting to note that the combination $e^{-\rho_{L}+\rho_{R}} \Omega_{L}^{+}\left(\Omega_{R}^{+}(\delta \Xi)\right)+e^{\rho_{R}} W_{1}+e^{-\rho_{L}} W_{2}$ resembles the holomorphic "three-form superfield" proposed in [18 and further discussed in [31. It is likely that the non-linearized version of this vertex operator should be written using pure spinors [32, which arise naturally in the description of generalized compactifications. 11

Let us see how $\left(W_{1}, W_{2}\right)$ appear in the full CFT. If the compactification manifold is an exact Calabi-Yau, the vertex operators $\Phi^{a}$ and $\Psi^{i}$ can be written as

$$
\Phi^{a}=\omega \frac{a}{I} \bar{\psi}_{L}^{\bar{I}} \psi_{R}^{J}, \quad \Psi^{i}=g_{K} h_{I} \frac{K}{J}_{\bar{\psi}} \bar{\psi}_{L}^{\bar{I}} \bar{\psi}_{R}^{\bar{J}}
$$

11 We would like to avoid a potential confusion with the pure spinor formalism of Berkovits [6]. However, although there is currently no explicit relation known between the Berkovits formalism and the pure spinors mentioned here, it is likely that they are intimately connected via the superconformal extension of the pure spinor string [5] compactified to four dimensions. 
where $\left(\psi_{L}, \psi_{R}\right)$ are the usual RNS fermions, $\omega_{I \bar{J}}^{a}$ is a harmonic $(1,1)$-form, $g_{I \bar{J}}$ is the Calabi-Yau metric and $h_{J}^{i \bar{K}}$ is an element of the Dolbeault cohomology group $H^{1,0}(\bar{T})$ of the Calabi-Yau. The introduction of fluxes deforms the original manifold and new cubic operators in the fermions

$$
\begin{aligned}
& \Upsilon=u_{I, \overline{J K}} \psi_{L}^{I} \bar{\psi}_{R}^{\bar{J}} \psi_{R}^{\bar{K}}, \quad \Theta=t_{I J, \bar{K}} \psi_{L}^{I} \psi_{L}^{J} \bar{\psi}_{R}^{\bar{K}}, \\
& \bar{\Upsilon}=\bar{u}_{\bar{I}, J K} \bar{\psi}_{L}^{\bar{I}} \psi_{R}^{J} \psi_{R}^{K}, \quad \bar{\Theta}=\bar{t}_{\overline{I J}, K} \bar{\psi}_{L}^{\bar{I}} \bar{\psi}_{L}^{\bar{J}} \psi_{R}^{K},
\end{aligned}
$$

should be included.12

We must now determine the relation between $\left(u_{I, \overline{J K}}, \bar{u}_{\bar{I}, J K}, t_{I J, \bar{K}}, \bar{t}_{\overline{I J}, K}\right)$ and the original physical deformations in terms of the RR fluxes. From (4.6) we can select the candidates for $\left(W_{1}, W_{2}\right)$ by counting charges and assuming that $(\Upsilon, \bar{\Upsilon}, \Theta, \bar{\Theta})$ are constant superfields in space-time;

$$
W_{1}=\Upsilon=u_{I, \overline{J K}} \psi_{L}^{I} \bar{\psi}_{R}^{\bar{J}} \bar{\psi}_{R}^{\bar{K}}, \quad W_{2}=\Theta=t_{I J, \bar{K}} \psi_{L}^{I} \psi_{L}^{J} \bar{\psi}_{R}^{\bar{K}}
$$

have the correct conformal weight. Substituting these into (4.4) we have

$$
\begin{gathered}
G_{L}^{+}\left(e^{\rho_{R}} u_{I, \overline{J K}} \psi_{L}^{I} \bar{\psi}_{R}^{\bar{J}} \bar{\psi}_{R}^{\bar{K}}+e^{-\rho_{L}} t_{I J, \bar{K}} \psi_{L}^{I} \psi_{L}^{J} \bar{\psi}_{R}^{\bar{K}}+\right. \\
\left.+e^{-\rho_{L}+\rho_{R}}\left(\theta_{L}-\theta_{R}\right)^{2} F_{a} \tilde{\omega}_{K M \overline{K M}}^{a} \psi_{L}^{M} \psi_{L}^{K} \bar{\psi}_{R}^{\bar{K}} \bar{\psi}_{R}^{M}\right) \rightarrow \\
\frac{e^{-\rho_{L}}}{z}\left(\partial_{M} t_{I J, \bar{K}}\right) \psi_{L}^{M} \psi_{L}^{I} \psi_{L}^{J} \bar{\psi}_{R}^{\bar{K}}+\frac{e^{\rho_{R}}}{z}\left(\partial_{M} u_{K, \overline{M K}}+F_{a} \tilde{\omega}_{K M}^{a} \overline{K M}\right) \psi_{L}^{M} \psi_{L}^{K} \bar{\psi}_{R}^{M} \bar{\psi}_{R}^{\bar{K}}=0
\end{gathered}
$$

where $\tilde{\omega}_{K M \overline{K M}}^{a}=\Omega_{M K}{ }^{\bar{I}} \bar{\Omega} \overline{M K}^{J} \omega_{\bar{I} J}^{a}$. Similarly, from the condition (4.5) we obtain

$$
\frac{e^{\rho_{R}}}{\bar{z}}\left(-\bar{\partial} \bar{M}_{I, \overline{J K}}\right) \psi_{L}^{I} \bar{\psi}_{R}^{\bar{M}} \bar{\psi}_{R}^{\bar{J}} \bar{\psi}_{R}^{\bar{K}}+\frac{e^{-\rho_{L}}}{\bar{z}}\left(\bar{\partial}_{\bar{M}} t_{K M, \bar{K}}+F_{a} \tilde{\omega}_{K M}^{a} \overline{K M}\right) \psi_{L}^{M} \psi_{L}^{K} \bar{\psi}_{R}^{\bar{M}} \bar{\psi}_{R}^{\bar{K}}=0
$$

Using $\bar{\partial}=d \bar{y}^{\bar{I}} \partial_{\bar{I}}$ and $\partial=d y^{I} \partial_{I}$ we can write these equations as

$$
\begin{gathered}
\partial t=\bar{\partial} u=0 \\
\partial u=\bar{\partial} t=-F_{a} \tilde{\omega}^{a}
\end{gathered}
$$

12 This choice is the most convenient for the discussion above. As in the case of the original chiral primaries, the cubic operators can be rotated using $\left(J_{L, R}^{++}, J_{L, R}^{--}\right)$. 
where $\tilde{\omega}^{a} \in H^{2,2}$ is the Hodge dual of $\omega^{a} \in H^{1,1}$ or, equvalently,

$$
d(t-u)=0 \quad, \quad d(t+u)=-2 F_{a} \tilde{\omega}^{a} .
$$

In the absence of flux $\Upsilon$ and $\Theta$ should be identified with $(s u(2)$ rotations of $)$ one of the original vertex operators $\Psi^{i}$. Note that since the value of $F_{a}$ is quantized there is no modulus corresponding to (4.3). In other words, it is a deformation of the $\sigma$-model preserving the full superconformal invariance but there is no massless space-time field corresponding to it. The next question to be addressed concerns how the presence of this vertex operator in the action affects the equations of motion for other modulus fields. One should expect that the equations of motion will show that with appropriate flux all the initial modulus fields will turn out to be massive.

From the discussion above we see that the correct vertex operators describing the presence of fluxes in the compactification-dependent sector have the general form

$$
\begin{gathered}
\mathcal{F}_{c c}=e^{-\rho_{L}} \Gamma+e^{-\rho_{R}} \Lambda+e^{-\rho_{L}-\rho_{R}} \Omega_{L}^{+}\left(\Omega_{R}^{-}\left(\Psi^{i}\right)\right)\left(\theta_{L}^{2} y_{i}+\bar{\theta}_{R}^{2} \bar{y}_{i}\right), \\
\mathcal{F}_{c a}=e^{-\rho_{L}} \Theta+e^{\rho_{R}} \Upsilon+e^{-\rho_{L}+\rho} \Omega_{L}^{+}\left(\Omega_{R}^{+}\left(\Phi^{a}\right)\right)\left(\theta_{L}^{2} F_{a}+\theta_{R}^{2} \bar{F}_{a}+\theta_{L} \theta_{R} D_{a}\right),
\end{gathered}
$$

where $\Gamma=g_{I, J K} \psi_{L}^{I} \psi_{R}^{J} \psi_{R}^{K}$ and $\Lambda=l_{I J, K} \psi_{L}^{I} \psi_{L}^{J} \psi_{R}^{K}$ are determined in terms of the fluxes $\left(y_{i}, \bar{y}_{i}\right)$ and the elements of $H^{1,0}(\bar{T}) .\left(\mathcal{F}_{c a}, \mathcal{F}_{c c}\right)$ are the operators corresponding to the superforms proposed in [18,31] up to the chiral boson dependence which implements the correct charges and conformal weights.

Now let us see how the presence of flux affects the vertex operator corresponding to space-time deformations. Since linearized fluctuations in $U$ can only describe perturbations satisfying $R_{\mu \nu}=0$, the effects of fluxes cannot be seen as coupled equations of RamondRamond operators and $U$ as in (4.10). One could try to include (4.10) into the action and re-compute physical state conditions. A more direct way is to compute UV divergences coming from interactions of $\mathcal{F}_{c a}$. For example, the composite operator $\mathcal{F}_{c a} \overline{\mathcal{F}}_{c a}$ has a UV divergence

$$
: \mathcal{F}_{c a}(z) \overline{\mathcal{F}}_{c a}(z):=\frac{1}{\epsilon^{2}} \theta_{L}^{\alpha} \theta_{R \alpha} \bar{\theta}_{L}^{\dot{\alpha}} \bar{\theta}_{R \dot{\alpha}} D_{a} D_{\bar{b}} \mathfrak{g}^{a \bar{b}}+\cdots
$$

where $\cdots$ contains terms with fewer $\theta \mathrm{s}, \mathfrak{g}^{a \bar{b}}$ is the Zamolodchikov metric 13 on the moduli space, and $\epsilon$ is a $\mathrm{UV}$ regulator in the $\mathrm{OPE}$

$$
\Phi^{a}(z) \bar{\Phi}^{\bar{b}}(w) \rightarrow \frac{\mathfrak{g}^{a \bar{b}}}{|z-w|^{2}+\epsilon^{2}} .
$$

13 This is one of the possible metrics. A second one can be defined as $\mathcal{G}_{L}^{+}\left(\mathcal{G}_{R}^{+}\left(\Phi^{a}\right)\right) \mathcal{G}_{L}^{-}\left(\mathcal{G}_{R}^{-}\left(\bar{\Phi}^{\bar{b}}\right)\right) \rightarrow$ $\frac{\mathfrak{G}^{a \bar{b}}}{|z-w|^{4}}$, and is the metric of a different section of the moduli space [23. 
This divergence breaks conformal invariance. Because of its $\theta$ dependence, (4.11) can only be cancelled by the vertex operator constructed from $U$ and since (4.10) does not break four-dimensional Poincaré invariance, this part of $U$ should be independent of $x$. As was mentioned in section 2.3, in a more general situation $U$ could be a function on the compactification manifold. At one loop (4.11) will be cancelled by : $T_{L} T_{R} U\left(\theta_{L}, \theta_{R}, \bar{\theta}_{L}, \bar{\theta}_{R}, y^{I}, \bar{y}^{\bar{J}}\right)$ :. This term is one of the many contributions from the integrated vertex operator (2.19). In a flat ten-dimensional background the divergence coming from this term is zero since it is proportional to $\square_{10} U$ which vanishes for a massless deformation. In the case at hand, where $U$ is independent of $x$, we have

$$
\begin{aligned}
& : T_{L} T_{R} U\left(\theta_{L}, \theta_{R}, \bar{\theta}_{L}, \bar{\theta}_{R}, y^{I}, \bar{y}^{\bar{J}}\right):+: \mathcal{F}_{c a}(z) \overline{\mathcal{F}}_{c a}(z):=
\end{aligned}
$$

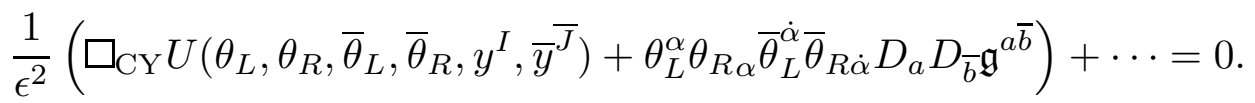

The component $\theta_{L}^{\alpha} \theta_{R \alpha} \bar{\theta}_{L}^{\dot{\alpha}} \bar{\theta}_{R \dot{\alpha}}$ is precisely where the space-time metric sits in $U$ (see (3.1)) and equation (4.12) implies the usual space-time warping in flux compactifications. In (4.12) $\cdots$ include other divergent terms that should also be cancelled, and this implies further corrections to the original background.

To compute superpotentials generated by fluxes in superstring scattering amplitudes we include (4.10) in the physical vertex operators (2.14) with appropriate $s u(2)$ rotations. For example, in the case of (4.1), there is a superpotential of the type

$$
\mathcal{W}=3 \int d^{4} x d^{2}\left(\theta_{L}-\theta_{R}\right) F_{a} M_{b} M_{c} \mathcal{K}^{a b c}
$$

where $d^{2}\left(\theta_{L}-\theta_{R}\right)$ is the measure for the preserved supersymmetry. Because of the mixing of $\left(\rho_{L}, \rho_{R}\right)$ and $\left(\mathcal{J}_{L}, \mathcal{J}_{R}\right)$ charges in $(4.10)$ and the zero-mode measure (2.23), the potential contributions from $\Theta$ and $\Upsilon$ do not appear and the presence of flux does not modify the topological amplitudes as argued by Vafa in [16]. It should be noted, however, that now $F_{a}$ is not interpreted as the auxiliary field in $M_{a}$, but a component of the physical operator $\mathcal{F}_{c a}$. To further study the supersymmetry breaking in this case, one can use the superspace projection technique introduced in Section 3. 


\section{Conclusions and Further Directions}

In this paper we analyzed the hybrid formalism for supersymmetry-breaking backgrounds. It was shown how worldsheet techniques can be used to study backgrounds with internal RR fluxes and a covariant formulation of the Type IIA string on $\left(\mathrm{CY}_{3} \times S^{1}\right) / \mathbb{Z}_{2}$ was constructed. The significant simplification of calculations in the hybrid formalism relative to the analogous ones in RNS is due to the absence of spin fields in the former. It was shown how the hybrid formalism can be applied to a much wider class of compactifications than the original $\mathrm{CY}_{3}$ case; much remains to be done in this direction.

There are many interesting applications that can follow from this work. For example, the hybrid is suitable for describing intersecting brane models which are currently one of the possible approaches to string phenomenology. Here the substitution of supercharges in favor of spin fields simplifies, among other things, the calculation of correlation functions associated to higher-dimension operators in the effective theory.

In a closely related line of research, one can use the hybrid formalism to study flux compactifications on Calabi-Yau orientifolds using a generalization of the superspace reduction technique of section 3. For example, in the Type IIA case, the inclusion of O6 planes projects out half of the spectrum by a superspace involution switching $\theta_{L} \leftrightarrow \theta_{R}$ in the case of chiral fields and $\theta_{L} \leftrightarrow \bar{\theta}_{R}$ in the case of twisted-chiral fields. Similarly to the analysis of section 4.1 , the surviving half-supersymmetric ${ }^{N} d={ }^{1} 4$ superfields are correlated with the induced projection on cohomology.

Many tools have been developed to study strings on Calabi-Yau spaces, like Gepner models [33], linear $\sigma$-models [34], and topological strings [35]. The presence of fluxes modifies the compactification CFT and such tools are no longer suitable. Since the main ingredient of these techniques is the $N=2$ supersymmetry algebra on the worldsheet and as we have shown that this symmetry is preserved, it is possible that there exist generalizations of these methods. For example, the $\widehat{c}=5$ formalism [17] has a linearly realized supersymmetry algebra on the worldsheet and one can add the space-time sector and a linear $\sigma$-model describing the internal space. One then searches for actions where the two algebras do not decouple. This would correspond to a generalized compactification. Another possibility is that topological strings on generalized complex spaces recently considered by Pestun in [36] will play an important role in future studies. Quantum corrections, both string loop and $\alpha^{\prime}$, should also be considered. Loop amplitudes of compactification-dependent states could be calculated in a supersymmetric way as in [25,37. In order to pursue this, one 
has to understand better the correlation functions of the time-like chiral boson $\rho$. To go beyond the linearized level, a general hybrid $\sigma$-model action with fluxes and warping can be constructed along the lines of [22]. This $\sigma$-model action is equivalent to the action of the uncompactified ten-dimensional superstring using $d=4 N=2$ notation. This would allow us to compute $\alpha^{\prime}$ corrections and consistency conditions for backgrounds with warping and internal RR flux using, for example, the beta function method. We hope to give simple examples of the hybrid formlism in this type of backgrounds such as $\mathrm{D} p$-brane solutions in the future.

Acknowledgments: We would like to thank Nathan Berkovits and Ram Sriharsha for discussions and comments. The work of BCV is supported by NSF grant number PHY 0354776 and the work of WDL3 is supported by NSF grant numbers PHY 0354776 and DMS 0502267. BCV also thanks University of North Carolina at Chapel Hill, where a part of this work was done, for their hospitality.

\section{Appendix A. Hybrid Compactified on $S^{1}$ : Supersymmetric Operators in $d=3$}

In this appendix we write, in a convenient way, the hybrid variables for the compactification Type II strings on $\mathrm{CY}_{3} \times S^{1}$. With the definitions of section 3 , the supersymmetric operators are

$$
\begin{gathered}
\Pi_{L}^{\alpha \beta}=\partial_{L} x^{\alpha \beta}+\theta_{L}^{(\alpha} \partial_{L} \bar{\theta}_{L}^{\beta)}+\bar{\theta}_{L}^{(\alpha} \partial_{L} \theta_{L}^{\beta)}, \quad Z=\partial_{L} x_{2}+\theta_{L}^{\alpha} \partial_{L} \bar{\theta}_{L \alpha}+\bar{\theta}_{L}^{\alpha} \partial_{L} \theta_{L \alpha} \\
d_{L \alpha}=p_{L \alpha}+\bar{\theta}_{L}^{\beta} \partial_{L} x_{\alpha \beta}-\frac{1}{2} \bar{\theta}^{2} \partial_{L} \theta_{\alpha}+\frac{1}{4} \theta_{L \alpha} \partial_{L} \bar{\theta}^{2}+\partial_{L} x_{2} \bar{\theta}_{\alpha}, \\
\bar{d}_{L \alpha}=\bar{p}_{L \alpha}+\theta_{L}^{\beta} \partial_{L} x_{\alpha \beta}-\frac{1}{2} \theta_{L}^{2} \partial_{L} \bar{\theta}_{L \alpha}+\frac{1}{4} \bar{\theta}_{L \alpha} \partial_{L} \bar{\theta}_{L}^{2}+\partial_{L} x_{2} \theta_{L \alpha},
\end{gathered}
$$

where $Z$ is a supersymmetric extension of the central charge operator $\partial_{L} x_{2}$. The algebra of these operators is

$$
\begin{gathered}
d_{L \alpha} \bar{d}_{L \beta} \rightarrow \frac{1}{z-w}\left(\Pi_{L \alpha \beta}+\epsilon_{\alpha \beta} Z\right), \quad d_{L \alpha} d_{L \beta} \rightarrow 0, \quad \bar{d}_{L \alpha} \bar{d}_{L \beta} \rightarrow 0 \\
d_{L \alpha} \Pi_{L \beta \gamma} \rightarrow \frac{1}{z-w} \epsilon_{\alpha(\beta} \partial_{L} \bar{\theta}_{\gamma)}, \quad \bar{d}_{L \alpha} \Pi_{L \beta \gamma} \rightarrow \frac{1}{z-w} \epsilon_{\alpha(\beta} \partial_{L} \theta_{L \gamma)} \\
d_{L \alpha} Z \rightarrow \frac{1}{z-w} \partial_{L} \bar{\theta}_{L \alpha}, \quad \bar{d}_{L \alpha} Z \rightarrow \frac{1}{z-w} \partial_{L} \theta_{L \alpha}
\end{gathered}
$$


With these definitions, the superconformal algebra is

$$
\begin{gathered}
T=\frac{1}{2} \Pi_{L \alpha \beta} \Pi_{L}^{\alpha \beta}+\frac{1}{2} Z_{L}^{2}+d_{L \alpha} \partial_{L} \theta_{L}^{\alpha}+\bar{d}_{L \alpha} \partial_{L} \bar{\theta}_{L}^{\alpha}+\mathcal{T}_{C Y} \\
G^{+}=e^{\rho_{L}} d^{2}+\mathcal{G}_{C Y}^{+}, \quad G^{-}=e^{-\rho_{L}} \bar{d}^{2}+\mathcal{G}_{C Y}^{-}, \quad J=-\partial_{L} \rho_{L}+\mathcal{J}_{C Y} .
\end{gathered}
$$

The spectrum is now characterized by the eigenvalues of the central charge operators if we compactify $x_{2}$ on a circle. Since there is left- and right-moving central charge, superfields are classified by two integers $(n, m)$ where $n$ is the Kaluza-Klein momentum and $m$ is the winding number.

Since we are breaking the full $\mathrm{SO}(1,3)$ covariance, we can define new super-covariant operators like $: d \bar{d}:, d \partial_{L} \bar{\theta}$, and $\bar{d} \partial_{L} \theta$. The algebra that is generated by these operators is a higher-spin algebra. It is likely that after breaking space-time and worldsheet supersymmetry these operators are related to the $G_{2}$ holonomy conformal algebra found by Shatashvili and Vafa [38] for a more general $G_{2}$ manifold than the special case considered here. 


\section{References}

[1] N. Berkovits, S. Gukov and B. C. Vallilo, Superstrings in 2D backgrounds with $R$ - $R$ flux and new extremal blackholes, Nucl. Phys. B 614, 195 (2001), arXiv:hep-th/0107140.

[2] N. Berkovits, Covariant Quantization of the Green-Schwarz Superstring in a CalabiYau Background, Nucl. Phys. B431, 258 (1994), arXiv:hep-th/9404162].

[3] N. Berkovits and C. Vafa, $N=4$ Topological Strings, Nucl. Phys. B433 (1995) 123, arXiv:hep-th/9407190.

[4] N. Berkovits, Quantization of the Type II Superstring in a Curved Six-Dimensional Background, Nucl. Phys. B565 (2000) 333, arXiv:hep-th/9908041.

[5] N. Berkovits, Pure spinor formalism as an $N=2$ topological string, JHEP 0510, 089 (2005), arXiv:hep-th/0509120.

[6] N. Berkovits, Super-Poincaré Covariant Quantization of the Superstring, JHEP 04 (2000) 018, arXiv:hep-th/0001035.

[7] K. Lee and W. Siegel, Conquest of the ghost pyramid of the superstring, JHEP 0508, 102 (2005) arXiv:hep-th/0506198.

[8] K. Lee and W. Siegel, Simpler superstring scattering, arXiv:hep-th/0603218.

[9] N. Berkovits, Review of open superstring field theory, arXiv:hep-th/0105230.

[10] N. Berkovits, The Ramond sector of open superstring field theory, JHEP 0111, 047 (2001) arXiv:hep-th/0109100.

[11] N. Arkani-Hamed, T. Gregoire and J. Wacker, Higher dimensional supersymmetry in $4 D$ superspace, JHEP 0203, 055 (2002) arXiv:hep-th/0101233.

[12] N. Berkovits, C. Vafa and E. Witten, Conformal Field Theory of AdS Background with Ramond-Ramond Flux, JHEP 9903 (1999) 018, arXiv:hep-th/9902098].

[13] N. Berkovits, M. Bershadsky, T. Hauer, S. Zhukov and B. Zwiebach, Superstring theory on $A d S(2) x S(2)$ as a coset supermanifold, Nucl. Phys. B 567, 61 (2000) arXiv:hepth/9907200].

[14] H. Ooguri and C. Vafa, The C-deformation of gluino and non-planar diagrams, Adv. Theor. Math. Phys. 7, 53 (2003) arXiv:hep-th/0302109.

[15] N. Seiberg, Noncommutative superspace, $N=1 / 2$ supersymmetry, field theory and string theory, JHEP 0306, 010 (2003) [arXiv:hep-th/0305248.

[16] C. Vafa, Superstrings and topological strings at large N, J. Math. Phys. 42, 2798 (2001) arXiv:hep-th/0008142.

[17] N. Berkovits, H. Ooguri and C. Vafa, On the worldsheet derivation of large $N$ dualities for the superstring, Commun. Math. Phys. 252, 259 (2004) arXiv:hep-th/0310118.

[18] A. Lawrence and J. McGreevy, Local string models of soft supersymmetry breaking, JHEP 0406, 007 (2004) [arXiv:hep-th/0401034].

[19] M. Grana, Flux compactifications in string theory: A comprehensive review, Phys. Rept. 423, 91 (2006) arXiv:hep-th/0509003]. 
[20] B. S. Acharya, On realising $N=1$ super Yang-Mills in $M$ theory, arXiv:hepth/0011089.

[21] R. Dijkgraaf, S. Gukov, A. Neitzke and C. Vafa, Topological M-theory as unification of form theories of gravity, arXiv:hep-th/0411073.

[22] N. Berkovits and W. Siegel, Superspace Effective Actions for $4 D$ Compactifications of Heterotic and Type II Superstrings, Nucl. Phys. B462 (1996) 213, arXiv:hepth/9510106].

[23] For a review, see L. J. Dixon, Some World Sheet Properties Of Superstring Compactifications, On Orbifolds And Otherwise, PUPT-1074, Lectures given at the 1987 ICTP Summer Workshop in High Energy Phsyics and Cosmology, Trieste, Italy, Jun 29 Aug 7, 1987; J. Distler, Notes on N=2 sigma models, arXiv:hep-th/9212062.

[24] N. Berkovits, Super-Poincare Invariant Koba-Nielsen Formulas for the Superstring, Phys. Lett. B 385, 109 (1996) arXiv:hep-th/9604120.

[25] N. Berkovits and B. C. Vallilo, One loop N-point superstring amplitudes with manifest $d=4$ supersymmetry, Nucl. Phys. B 624, 45 (2002) arXiv:hep-th/0110168.

[26] N. Marcus, A. Sagnotti and W. Siegel, Ten-Dimensional Supersymmetric Yang-Mills Theory In Terms Of Four-Dimensional Superfields, Nucl. Phys. B 224, 159 (1983).

[27] D. D. Joyce. Compact Manifolds with Special Holonomy. Oxford Science Publications, Oxford, UK, (2000).

[28] G. Papadopoulos and P. K. Townsend, Compactification of D = 11 supergravity on spaces of exceptional holonomy, Phys. Lett. B 357, 300 (1995) arXiv:hep-th/9506150.

[29] R. Roiban and J. Walcher, "Rational Conformal Field Theories With G(2) Holonomy," JHEP 0112, 008 (2001) arXiv:hep-th/0110302.

[30] T. R. Taylor and C. Vafa, RR flux on Calabi-Yau and partial supersymmetry breaking, Phys. Lett. B 474, 130 (2000) arXiv:hep-th/9912152].

[31] M. Grana, R. Minasian, M. Petrini and A. Tomasiello, Supersymmetric backgrounds from generalized Calabi-Yau manifolds, JHEP 0408, 046 (2004) arXiv:hepth/0406137].

[32] M. Gualtieri, Generalized complex geometry, arXiv:math.dg/0401221.

[33] D. Gepner, Exactly Solvable String Compactifications On Manifolds Of SU(N) Holonomy, Phys. Lett. B 199, 380 (1987).

[34] E. Witten, Phases of $N=2$ theories in two dimensions, Nucl. Phys. B 403, 159 (1993) arXiv:hep-th/9301042.

[35] A. Neitzke and C. Vafa, Topological strings and their physical applications, arXiv:hepth/0410178.

[36] V. Pestun, Topological strings in generalized complex space, arXiv:hep-th/0603145.

[37] J. Kappeli, S. Theisen and P. Vanhove, A note on topological amplitudes in hybrid string theory, arXiv:hep-th/0607021.

[38] S. L. Shatashvili and C. Vafa, Superstrings and manifold of exceptional holonomy, Selecta Math. 1, 347 (1995) [arXiv:hep-th/9407025]. 